\title{
BETRACHTERPERSPEKTIVEN IM KONFLIKT
}

\author{
Zur Überlieferungsgeschichte der „vecchiarella“-Anekdote
}

\author{
Felix Tbürlemann
}

\section{Von der Künstleranekdote zur Betrachteranek- dote}

Im Vorwort zu der 1646 in Rom veröffentlichten Sammlung von Stichen nach Carracci-Zeichnungen berichtet Giovanni Antonio Massani (unter dem Pseudonym Giovanni Atanasio Mosini) zum erstenmal eine Anekdote aus dem Leben Annibale Carraccis, die in der Kunstliteratur des nachfolgenden Jahrhunderts immer wieder nacherzählt, umgeformt und kritisch besprochen wird. ${ }^{1}$ Nach der Figur eines alten Weibleins, das darin die Rolle einer Kunstrichterin über die Werke zweier Schüler Annibales spielt, pflegt man sie heute als „vecchiarella“-Anekdote zu bezeichnen. Massani nennt die Namen der beiden Maler und den Standort ihrer Werke nicht, so daß bei ihm die Anekdote gleichzeitig einem Rätsel gleichkommt. In wörtlicher Übersetzung lautet sie wie folgt (vgl. Anhang, Text 1, ohne Einleitung):

„Einer der besten Literaten jener Zeit stellte Annibale einmal die Frage, welcher von zwei Malern aus seiner Schule sich besser angestell thabe bei einer Arbeit, die sie zusammen für einen Kardinal ausgeführt hatten: [Es handelt sich] für jeden der beiden [um] eine grosse Historie aus dem Leben eines gleichen Heiligen, die in einer Kirche Roms in Freskotechnik auf die zwei Seitenwände, einander gegenüber gemalt sind. Auf diese Frage antwortete Annibale, die beiden Historien seien für ihn der Grund gewesen, daß er sich als ein riesiger Tölpel vorgekommen sei; denn er habe nie begreifen können, welche von ihnen mehr Lob verdiene, bis er es von einer alten Frau zu verstehen gelernt habe. Mit einem kleinen Mädchen an der Hand blieb diese eines Tages stehen, um jede der beiden Historien zu betrachten, und er beobachtete sie dabei: Während sie den Blick auf die eine [der beiden Historien] richtete, liess sie das Auge nach allen Seiten hin schweifen, um sie ganz zu beschauen; sie sagte aber nie ein Wort, noch gab sie sonst ein Zeichen irgend einer Gemütsbewegung, welche das Betrachten jenes Gemäldes bei ihr hervorgerufen hätte. Doch dann, nachdem sie sich zur anderen Historie hingewandt hatte, begann sie zum Mädchen zu sagen: 'Schau, schau, mein Töchterchen, diesen Mann, der sowas tut', und zeigte ihm mit dem Finger die Figur, welche die Handlung, die sie benannte, darstellte. Und während sie so die übrigen Figuren eine nach der andern betrachtete, wies sie [jedesmal] mit dem Finger auf sie hin, und mit Vergnügen erklärte sie deren Handlungen dem Mädchen, das sich ebenfalls daran zu ergötzen schien. 'Jetzt seht ihr', sagte Annibale zum Literaten, 'wie ich zu verstehen gelernt habe, welcher von unseren beiden Malern die Affekte lebendiger ausgedrückt und seine Geschichte klarer dargelegt hat.' Und dies genügte als eine sehr klare Antwort auf jene Frage."

Der zeitgenössische Leser dürfte keine Schwierigkeiten gehabt haben, die von Massani verschwiegenen Namen zu ergänzen: Die beiden Künstler waren Guido Reni und Domenichino, ihre Werke zwei Szenen aus dem Leben des Apostels Andreas, mit denen sie 1609 im Auftrag des Kardinals Scipio Borghese das dem Heiligen geweihte Oratorium auf dem Celio ausgemalt hatten. Der Leser war aufgefordert, die in der Anekdote vorgelegten Beurteilungskriterien gegenüber den Werken zu prüfen, mit dem Ziel, das gemeinte bessere Bild zu identifizieren.

Massani verschweigt die Namen der beiden Maler wohl vor allem deshalb, damit die Anekdote den Charakter einer primär Annibale betreffenden Geschichte bewahren kann; als ein Beispiel für seine Schlagfertigkeit, als ein ,inditio della finezza dell'ingegno del Carracci“ wird sie angeführt. Anders steht es mit allen späteren Wiedergaben der Anekdote. Sie dient dort ausschließlich dazu, die Werke Domenichinos und Renis im Sinne des 
Paragone gegeneinander auszuspielen - entsprechend tritt die Person Annibales in den Hintergrund. Ursprünglich als Künstleranekdote erzählt nimmt die Geschichte in der Folge ganz den Charakter einer Betrachteranekdote an.

\section{Problemstellung}

Die Unterscheidung zwischen Künstler- und Betrachteranekdote ist in der kunstwissenschaftlichen Literatur nicht üblich. Die Anekdote als „knappe Darstellung einer merkwürdigen Begebenheit" ${ }^{\text {"2 }}$ kann jedoch als zentralen Handlungsträger gleichermaßen beide Akteure der künstlerischen Kommunikation, den Produzenten und den Rezipienten fungieren lassen, auch wenn das stärkere Interesse an der Figur des schöpferischen Individuums die Bildung der Künstleranekdote im engeren Sinne begünstigt. Im Unterschied zu Ernst Kris und Otto Kurz, die in ihrer bekannten Studie „Die Legende vom Künstler" die Künstleranekdoten mit dem Ziel untersucht haben, die „Vorstellung vom Künstler" zu rekonstruieren ${ }^{3}$, beschäftigen wir uns hier mit der Betrachteranekdote. ${ }^{4}$ Aber auch unser methodischer Ansatz ist ein anderer. Während Kris und Kurz die ,typischen Züge in der Biographik vom bildenden Künstler" ${ }^{* 5}$ anhand der ganzen europäischen und fernöstlichen Kunstliteratur behandelt haben, soll im folgenden eine einzige Anekdote in ihren verschiedenen Versionen den Gegenstand der Untersuchung bilden. Unser Interesse gilt nicht den invarianten Motiven des Anekdotenschatzes, sondern den unterschiedlichen Darstellungsformen einer ausgewählten Anekdote. ${ }^{6}$

Die Kunstwissenschaft ist sich in den letzten Jahren immer stärker des Ungenügens einer rein stilgeschichtlichen Betrachtungsweise bewußt geworden. Man hat erkannt, daß nicht nur der Produktionsprozeß mit seinen stilistischen und ikonographischen Darstellungskonventionen, sondern auch der Rezeptionsprozeß dem geschichtlichen Wandel unterworfen ist. Die kunstwissenschaftliche Analyse setzt als zusätzliche Aufgabe die Rekonstruktion einer dem Werk adäquaten $B e$ trachterkompetenz voraus. ${ }^{7}$ Zur Erfüllung dieser Aufgabe stehen grundsätzlich zwei Wege offen: die Analyse der kunsttheoretischen Literatur im wei- testen Sinne - wozu auch die Betrachteranekdoten zu zählen sind - und die Analyse der Werke selber, d.h. der bildeigenen Mittel, mit denen der Künstler den Bezug zur Welt des Betrachters gestaltet. ${ }^{8}$ Die Art und Weise, wie in einem gegebenen Werk das Verhältnis zwischen Bildwelt und Betrachterwelt artikuliert wird, nennen wir die Betrachterperspektive. ${ }^{9}$

Für die nachfolgende Untersuchung gehen wir von der These aus, daß die Analyse der Betrachterperspektive wesentlich zur Charakterisierung einer ästhetischen Konzeption beitragen kann. Dabei ist zu beachten, daß zu einer gegebenen kulturellen Epoche gleichzeitig unterschiedliche oder gar gegensätzliche Betrachterperspektiven Gültigkeit haben können. Die Überlieferungsgeschichte der „vecchiarella“-Anekdote legt - wie wir sehen werden - von einer solchen konfliktuellen Situation Zeugnis ab.

Der vorliegenden Analyse der "vecchiarella“Anekdote liegt folgende Fragestellung zugrunde: Unter welchen Bedingungen kann die Betrachteranekdote zur Rekonstruktion der Betrachterperspektive und damit zur Werkinterpretation beitragen? Wir werden uns also nicht darauf beschränken, die der Anekdote eigenen ästhetischen Konzepte zu explizieren, sondern den Text auf die beiden Werke, die er betrifft, zurückbeziehen. Im Falle der ,vecchiarella“-Anekdote drängt sich eine solche Rückbeziehung geradezu auf. Es besteht nämlich eine merkwürdige, in der neueren kunstwissenschaftlichen Literatur nicht beachtete strukturelle Analogie zwischen der „vecchiarella“Anekdote einerseits und den Fresken Renis und Domenichinos andererseits (Abb. 4, 5). Die zwei Akteure Frau und Kind, die in der Anekdote als Betrachter der Bilder auftreten, nehmen in den beiden Bildern selber jeweils eine prominente Stellung ein, und zwar ebenfalls in der Rolle von Betrachtern gegenüber der dargestellten Geschichte. Auf Grund dieses besonderen Verhältnisses zum Werk wird die „vecchiarella“-Anekdote auch als Ausgangspunkt für allgemeine Überlegungen zur Funktion des Mutter-Kind-Motivs in der Historienmalerei dienen können. ${ }^{10}$ 


\section{Ein Dispositiv der Wahl}

Begeben wir uns in der Folge der "vecchiarella“ und Annibale Carraccis auf den Celio zur Kirche San Gregorio Magno. Vor der Fassade führt links eine Treppe hinauf zu einem abgetrennten Bezirk mit drei, nach einem axialsymmetrischen Schema angeordneten Kapellen (Abb. 1, 2). Die mittlere, durch einen Portikus ausgezeichnet, ist das Oratorio di S. Andrea. Im Kapelleninnern (Abb. 3) erblickt man als dominierenden malerischen Schmuck auf der rechten Seitenwand die Szene der Geißelung des Titelheiligen, das Werk Domenichinos (Abb. 5), und auf der linken Seitenwand, von der Hand Guido Renis, die Darstellung seines Ganges zur Kreuzigung (Abb. 4). ${ }^{11}$

Bereits die räumliche Ausgangslage als solche ist bedeutungsvoll. Sie bringt die beiden Fresken potentiell in ein Verhältnis des Gegensatzes zueinander. Wegen der - durch die mittlere Position der Andreas-Kapelle unterstrichenen - axialsymmetrischen Anordnung stehen sie sich als vergleichbare, gleichwertige Elemente gegenüber. Doch mehr noch als eine Anregung zum Vergleich ist dieses Dispositiv eine Aufforderung zur Wabl: Wer die Kapelle betritt, kann die beiden Fresken nicht aus der gleichen Position heraus betrachten; er muß sich zum einen oder anderen hinwenden. In der räumlichen Anordnung der beiden Werke liegt somit - zusammen mit dem Wissen, daß sie von zwei verschiedenen Künstlern stammen - das eigentliche auslösende Moment der „vecchiarella“Anekdote. Dies hat schon Passeri klar erkannt. Er sieht in der frontalen Gegenüberstellung den Grund, weshalb sich täglich von neuem die seines Erachtens sinnlose Frage "Welches ist das bessere der beiden Werke?" entfacht: „Quest'opera [das Fresko Domenichinos] perche sta à fronte ad una di Guido, fa nascere giornalmente delle contentioni ..."12

Es ist jedoch zu beachten, daß dieses von Passeri kritisierte Gegeneinanderausspielen von Malern der gleichen Epoche gerade in der Kunstliteratur des 17. Jahrhunderts einen beinahe obsessionellen Stellenwert einnimmt. Da die Geschichte der Künste in Übernahme eines antiken Darstellungsmusters als Weg einer beständigen Verbesserung („perfezionamento“) mit möglichen Rückschlägen betrachtet wurde, stellte sich notwendigerweise die

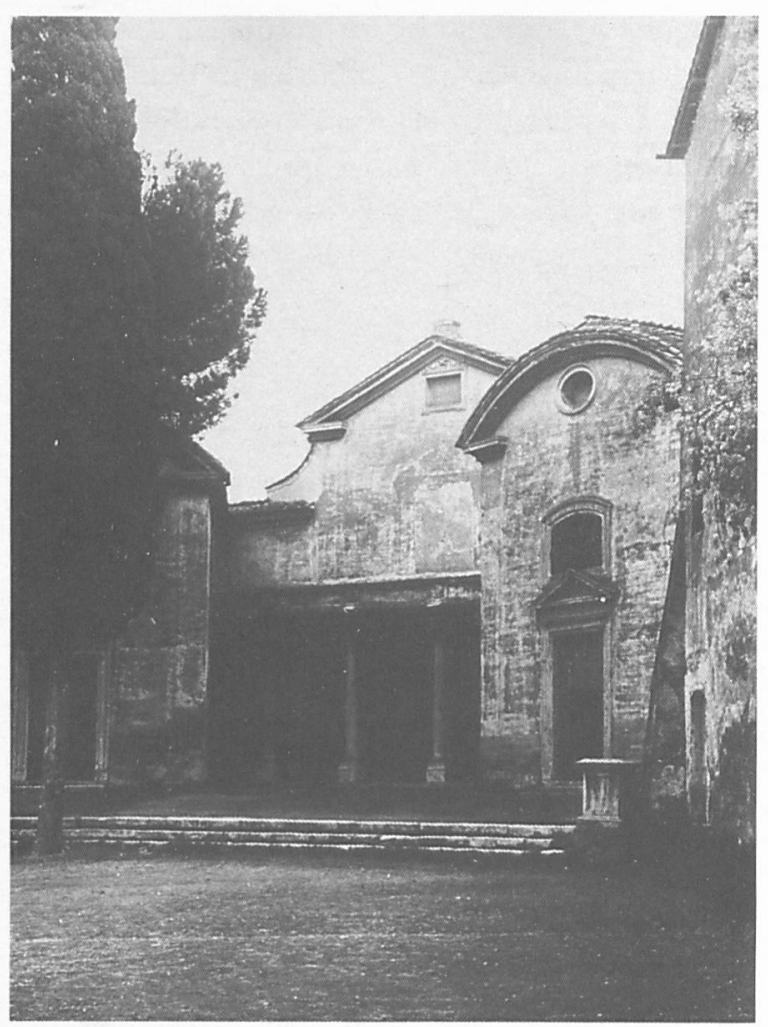

1. Oratorien der hl. Barbara, Andreas und Silvia bei S. Gregorio Magno, Rom

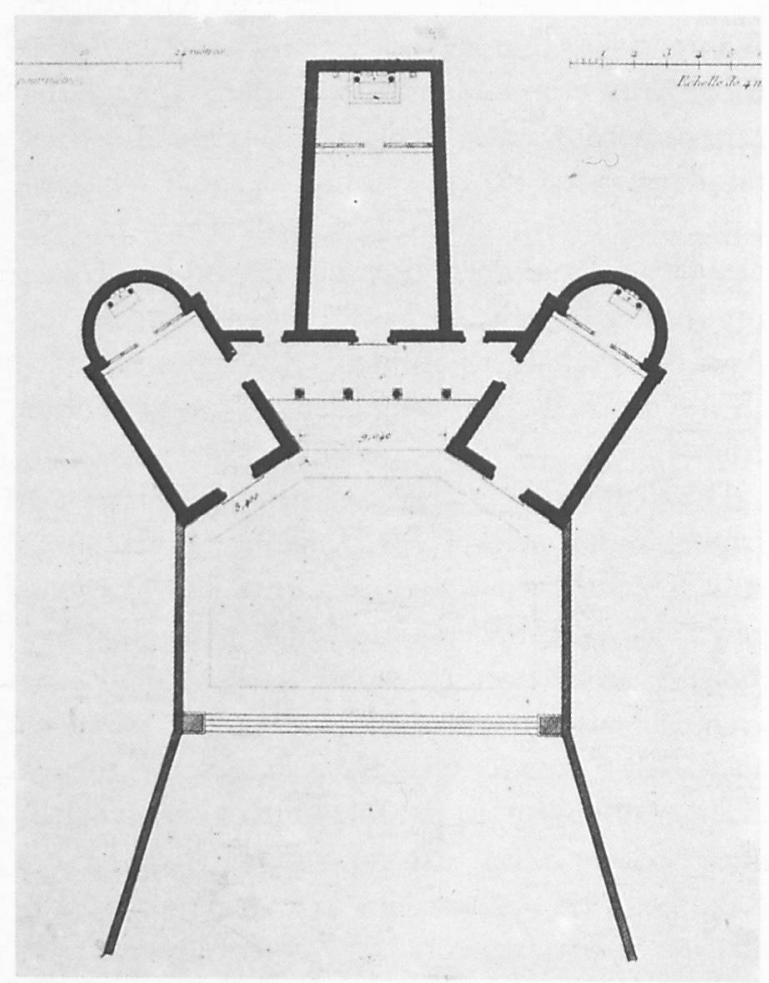

2. Grundriß der drei Oratorien nach Letarouilly, Edifices de Rome moderne, vol. II, Paris 1868 


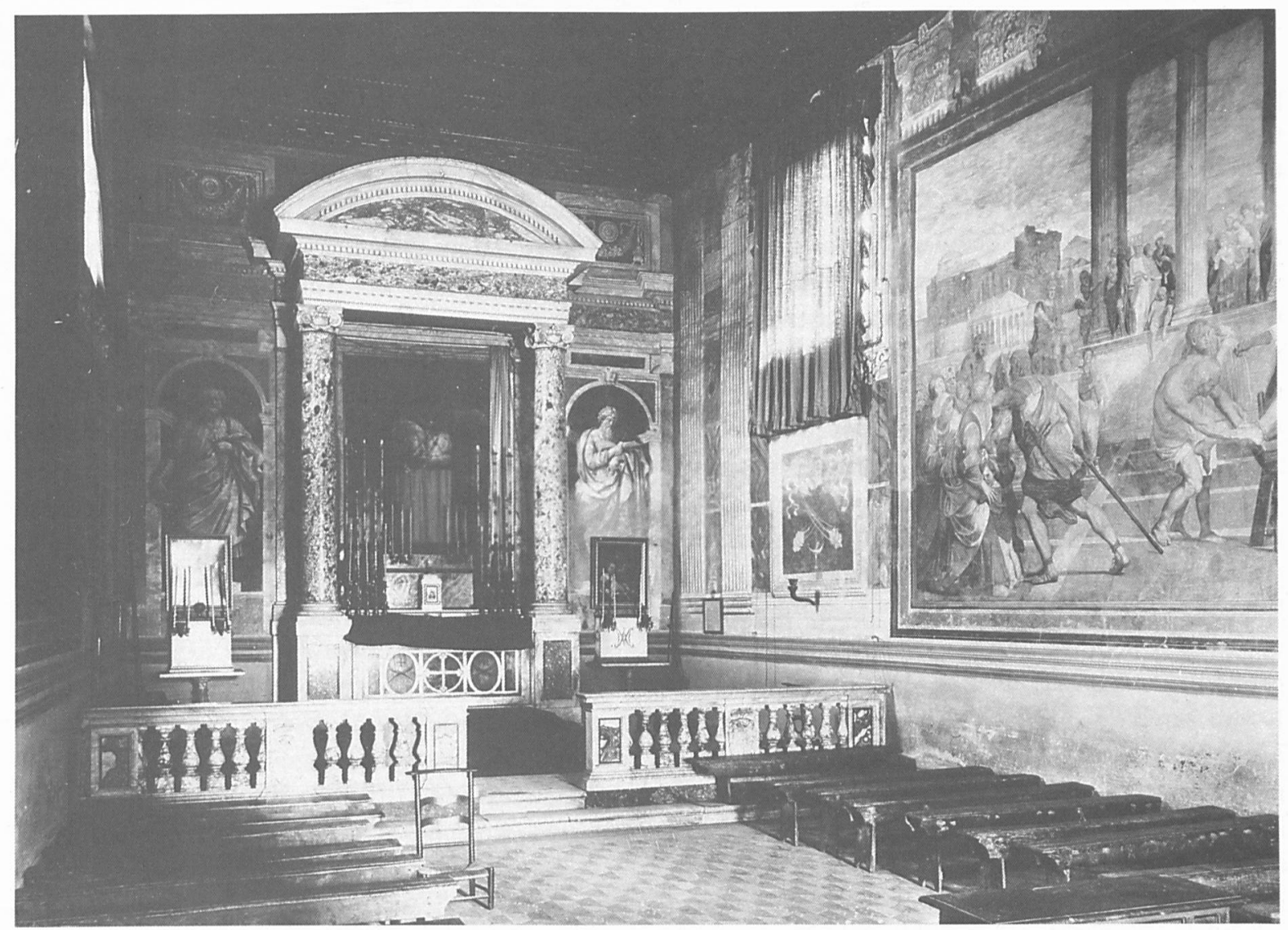

3. Rom, Oratorio di S. Andrea. Innenansicht

Frage, welcher Künstler mit seinem Schaffen jeweils den höchsten Stand der Kunst erreicht habe. ${ }^{13}$ So gibt z.B. Bellori in der Vita Annibale Carraccis gleich hintereinander die Antworten wieder, welche der Maler angeblich auf die Fragen nach der jeweiligen Vorrangstellung für die folgenden drei Künstlerpaare gegeben hat: Raffael und Tizian, Tasso und Ariost sowie - wiederum - Reni und Domenichino. ${ }^{14}$

Vor die Wahl gestellt gegenüber den Fresken Renis und Domenichinos waren bald auch die angehenden Maler Roms, die in großer Zahl zum Oratorio di S. Andrea pilgerten, um nach ihnen zu zeichnen. Die Kapelle wurde für sie zu einem Ort des ästhetischen Bekenntnisses, wie Félibien in seiner Biographie Poussins zu berichten weiß:

"C'estoit dans le temps que la pluspart des jeunes Peintres qui estoient à Rome, attirez par la grande réputation où estoit le Guide, alloient avec empressement copier son tableau du Martyr de Saint
André qui est à Saint Gregoire. Le Poussin estoit presque le seul qui s'attachoit à dessiner celuy du Dominiquin, lequel est dans le mesme endroit; \& il en fit si bien remarquer la beauté, que la pluspart des autres Peintres, persuadez par ses paroles \& par son exemple, quitterent le Guide pour étudier d'après le Dominiquin. “15

Es ist unwahrscheinlich, daß die Werke Domenichinos und Renis im Zeichen feindlicher Rivalität entstanden sein könnten. Dagegen spricht die persönliche Freundschaft, die in den frühen Jahren zwischen ihnen bestand. ${ }^{16}$ Umso interessanter ist es zu sehen, wie das Paragonedenken, unterstützt durch die räumliche Disposition der beiden Fresken, die kunstkritische Betrachtung immer stärker auf deren gestalterische Differenzen gelenkt und bisweilen zur Annahme eines Konkurrenzverhältnisses zwischen den Malern geführt hat.

Hier ist eine Zwischenbemerkung angebracht. In der Gegenüberstellung der beiden Fresken 
Renis und Domenichinos scheint auf den ersten Blick die typische Situation des induktiven Vergleichs vorzuliegen. Dieses Vorgehen, das methodisch bisher kaum hinterfragt worden ist, wird noch heute als heuristisches Mittel in der kunstgeschichtlichen Forschung und Lehre häufig eingesetzt. ${ }^{17}$ Es besteht darin, zwei Werke, die irgendwelche gemeinsamen ikonographischen oder formalen Merkmale aufweisen, zusammenzustellen und auf zusätzliche Ähnlichkeiten und Unterschiede zu befragen. Dabei wird von der Erwartung ausgegangen, es ließe sich so das "Wesen“ der verglichenen Werke bzw. der Kunst ihrer Autoren genauer fassen. Daß dieses Vorgehen, wenn es nicht von den Prinzipien einer übergreifenden Fragestellung geleitet wird, problematisch ist, wird aus folgender Tatsache ersichtlich: Je nach Vergleichsobjekt, mit dem ein bestimmtes Werk zusammengebracht wird, ergeben sich unterschiedliche Resultate, die leicht zu widersprüchlichen Charakterisierungen des gleichen Ausgangsobjektes führen können. ${ }^{18}$

In der ,vecchiarella“-Anekdote geht es jedoch nicht um eine differentielle stilistische Charakterisierung in der Art der neueren Kunstgeschichtsschreibung. ${ }^{19}$ Wiederum ganz entsprechend dem Prinzip des „perfezionamento“ der Malerei werden die Werke zweier "Schüler" des gleichen Meisters in Bezug auf eine bestimte Aufgabe, die wirksame Darstellung der Historie, befragt, wobei der Meister selber aufgefordert ist, das Urteil zu fällen. ${ }^{20}$

\section{Die Erstfassung der Anekdote}

Betrachten wir die "vecchiarella“-Anekdote nochmals in der ältesten, von Massani berichteten Fassung (Anhang, Text 1), wie sie direkt oder indirekt für alle späteren Schilderungen als Ausgangspunkt dienen wird. Wie bereits bemerkt, führt Massani die Anekdote als ein Beispiel für die Schlagfertigkeit Annibales ("galanteria [...] di parole", "finezza dell'ingegno") an. Die Art und Weise, wie die Geschichte eingeleitet wird ("un modo da lui tenuto, per lodare più uno, che un'altro di due suoi scolari"), deutet darauf hin, $\mathrm{da}$ Massani in erster Linie Annibales raffinierten Dreh bewundert, wie dieser durch das Vorschieben der „vecchiarella“ über die beiden Maler ein Urteil abgeben kann, ohne sich dabei selber exponieren zu müssen. ${ }^{21}$

Untersucht man jedoch die Antwort Annibales genauer, zeigt sich, daß ihr eine viel weitergehende Bedeutung zukommt. Sie berichtet, wie Annibale die ihm anfänglich fehlenden Kriterien zur Beurteilung der Werke erwirbt. Daß der Kern der Geschichte, d.h. die narrative Transformation, diesen Aspekt betrifft, wird über den Vergleich der parallel gestalteten Ausdrücke deutlich, welche Ausgangs- und Endsituation schildern: Dem einleitenden "non haveva mai saputo comprendere, quale, di esse meritasse d'esser più lodata" steht am Schluß der Satz gegenüber: „Hor vedete [...] com'io hò imparato à conoscere, quale delli nostri due Dipintori habbia più vivamente espressi gli affetti, e più chiaramente la sua Historia dichiarata“". Der Maler, der größeres Lob verdient - dies hat die Reaktion der Alten den Annibale gelehrt ist jener, der die Affekte auf lebendigere Art ausdrückt und seine (d.h. die ihm als Vorwurf dienende) Geschichte auf deutlichere Weise erläutert.

Die beiden Kriterien betreffen zwei zentrale Aspekte der seicentesken Theorie der Historienmalerei. Ebenfalls zwei Elemente kommen in der Reaktion der "vecchiarella“ und des Mädchens ins Spiel: das affektive Berübrtsein und das Sprechen. Sie bilden auf der Rezeptionsseite das Komplement zu den beiden Kriterien, mit denen die Schöpfungen der Maler beurteilt werden. Während nach einer eingehenden Beobachtung von Renis Fresko bei der Alten eine Reaktion im zweifachen Sinne ausbleibt (,non disse mai una parola, nè diede altro segno d'alcun affetto"), wird beim Anblick von Domenichinos Fresko unmittelbar eine starke positive Reaktion registriert. Die Beschreibung dieser Reaktion durch Massani kommt einer äußerst aufschlußreichen Darstellung des Rezeptionsprozesses gleich. Die Wahrnehmung des Bildes wird primär als Sprechen konzipiert: Die Alte erläutert dem sie begleitenden Mädchen das Bild mit sprachlichen Mitteln. Wirkungsvoll ist das Historienbild offenbar dann, wenn es die Übersetzung bzw. Rückübersetzung in die sprachlich geformte Geschichte erlaubt. Wenn in den Traktaten des 16. und 17. Jahrhunderts in Umkehrung des Prinzips 'ut pictura poesis' das Bild als stummes Buch ("libro muto e taciturno") bezeichnet wird ${ }^{22}$, 
besteht die Rezeption des Bildes entsprechend darin, diesem die fehlende Stimme zurückzugeben. Was die „vecchiarella“ dem Mädchen gegenüber formuliert, ist die Aussage des Bildes, gleichsam das, was es sagen würde, wenn es sprechen könnte. Wir erinnern uns, daß eines der von Annibale erworbenen Beurteilungskriterien „chiaramente la sua Historia dichiarare" hieß. Das gleiche Verb wird für die Tätigkeit der Alten verwendet: „dichiarava [...] le attioni“. Es ist also das Bild selber, das, indem es die "vecchiarella" zum Sprechen bringt, seinen Inhalt, die Geschichte, öffentlich und deutlich ausspricht. ${ }^{23}$

Der Vorgang der Rezeption besteht im sukzessiven Aufbau der Geschichte, ausgehend von der Erfassung und Identifikation der Handlungsmomente der einzelnen Figuren. Das Ergebnis käme im vorliegenden Fall einer umfassenden analytischen Beschreibung des Handlungsverlaufes gleich, wenn diese von Massani nicht unterdrückt worden wäre. Eine solche analytische Darstellung findet sich jedoch bei Bellori (Anhang, Text 3). Dieser gibt vorgängig eine eigene Schilderung von Domenichinos "Geißelung des hl. Andreas"; darauf berichtet er die Anekdote - offenbar im Anschluß an Massani - und fügt darin eine nochmalige Beschreibung des Bildes ein. Diese zweite, der "vecchiarella“ in den Mund gelegte Beschreibung erscheint zwar deutlich reduziert im Verhältnis zur ersten (sie beschränkt sich auf die Martyriumsszene der rechten Seite und erwähnt nur deren vier wichtigste Akteure), beide folgen jedoch demselben Prinzip. In beiden wird „figura per figura" vorgegangen, wobei zu jeder Figur Handlungselement und Affekt getrennt aufgeführt werden. ${ }^{24}$

Die Beurteilungskriterien, „vivamente esprimere gli affetti“ und „chiaramente la sua Historia dichiarare", die Annibale aus der doppelten Reaktion der Alten gewonnen hat, können zwei der drei Funktionen zugeordnet werden, welche nach der antiken Rhetorik die Rede zu erfüllen hat: movere und docere. Doch auch die dritte Aufgabe der Rede, das delectare, wird zwar nicht explizit als Beurteilungskriterium des Bildes formuliert, ist aber in Massanis Darstellung des Rezeptionsprozesses deutlich greifbar. Von der "vecchiarella“ heißt es: "dichiarava con gusto le attioni“, von der „fanciulla": „pareva che se ne prendesse diletto". So lassen sich in Massanis Schilderung der Anekdote alle drei Funktionen nachweisen, die Paleotti in seinem „Discorso intorno alle imagini sacre e profane" in Analogie zur Redekunst für die Malerei postuliert:

"Quello [...] che abbiamo detto chiamarsi ufficio del pittore [...] pare a noi che da nissun altro luogo meglio si possa cogliere, che dalla stessa comparazione degli scrittori, a' quali per ufficio dell'arte è imposto che debbano dilettare, insegnare e movere. Parimente dunque ufficcio del pittore sarà usare li stessi mezzi nella sua opera, faticandosi per formarla di maniera, che ella sia atta a dare diletto, ad insegnare e movere l'affetto di chi la guarderà. “ ${ }^{25}$

Schließlich läßt sich auch die Wahl von Frau und Kind als Betrachterfiguren mit der gegenreformatorischen Theorie des Historienbildes in Beziehung bringen. Paleotti und alle übrigen Traktatisten der Gegenreformation knüpfen für ihre Darlegungen bei den Kirchenvätern an. Diese rechtfertigen die Präsenz der Bilder in den Kirchen (gegenüber der ikonoklastischen Bewegung) bekanntlich mit dem Hinweis auf die Leistung, welche das Bild als Bibel für die idiotae, die des Lesens Unkundigen, erfüllen könne. Am häufigsten wird in den Traktaten eine Stelle aus einem Brief Gregors des Großen angeführt:

„[...] quod legentibus scriptura, hoc idiotis praestat pictura cernentibus, quia in ipsa ignorantes vident, quod sequi debeant, in ipsa legunt qui litteras nesciunt; unde praecipue gentibus pro lectione pictura est. ${ }^{\text {26 }}$

Aus der vorangegangenen Analyse geht hervor, daß die "vecchiarella"-Anekdote in der von Massani überlieferten Fassung einige wesentliche Elemente der gegenreformatorischen Theorie des Historienbildes wie durch ein Brennglas gebündelt darstellt. Die Tatsache, daß die Anekdote in klar artikulierter Form eine bestimmte ästhetische Position vertritt, ist auch der Grund, weshalb sie in der Kunstliteratur des späten 17. und frühen 18. Jahrhunderts zu einem beständigen Gegenstand der kritischen Auseinandersetzung wird. ${ }^{27}$ 


\section{Variationen über die Anekdote}

Die Auffassung des Bildes als Schriftersatz für die des Lesens Unkundigen - nicht nur Kinder, auch ein Großteil der Frauen waren dies bekanntlich im 17. Jahrhundert - ließ sich zwar mit dem von der klassizistischen Kunsttheorie zum Leitspruch erhobenen 'ut pictura poesis' gut vereinbaren; sie kam aber gleichzeitig in einen unauflösbaren Widerspruch zu ihrer idealistischen Grundtendenz. Agucchi und Bellori etwa werden nicht müde, den "plebe“, der bloß für oberflächliche Sinnesreize empfänglich sei, als Kunstrichter zu disqualifizieren. Die große Kunst richtet sich nach Agucchi an den "uomo intendente" ${ }^{28}$ Es ist klar, daß die alte Frau und das Kind in der Anekdote als exemplarische Bildbetrachter mit einer bestimmten demonstrativen Funktion aufzufassen sind; sie dürfen keinesfalls als repräsentative Darstellung des Bildbetrachters im soziologischen Sinne verstanden werden. Dies wird deutlich bei Bellori, der die „vecchiarella“-Anekdote gerade deshalb anführt, um Domenichino vom Standpunkt seines elitären Klassizismus aus gegenüber Reni zu verteidigen, der - wie er selber bemerkt - „von jedermann wegen der Gefälligkeit und Lieblichkeit seines Pinsels" bevorzugt werde. ${ }^{29}$ So zieht Alois Riegl in der posthum erschienenen Vorlesung „Die Entstehung der Barockkunst in Rom" aus einer direkten, kontextlosen Lektüre gerade die verkehrte Schlußfolgerung aus der Anekdote: „Das will also sagen: Domenichino malte das, was das Volk wollte und sprach zum Volke. Guido malte für Feinschmecker" ${ }^{30}$

Es ist Passeri, der in seinen zu Lebzeiten ungedruckten Künstlerbiographien den Widerspruch zwischen der Anekdote und der klassizistischen Kunsttheorie sichtbar macht und den Wert der Berufung auf die Reaktion der "vecchiarella" - er nennt sie „stolida“ (töricht) - im Streit um die beiden Fresken radikal in Frage stellt; gleichzeitig unterstreicht er die hohen intellektuellen Anforderungen, die ein vergleichendes Qualitätsurteil stelle:

„Non basta il senso dell'occhio con la sodisfatione della vista; e quello dell'orecchio, con appagarsi d'haver inteso dire; se l'intelletto non concorre in aiuto con un perfetto conoscimento, appoggiato ad uno scientiato sapere. Quel pro- porre, o negare la semplicità d'una stolida Vecchiarella non è autorità così valida, che possa stabilire il fondamento d'una machina d'inalzarvi la sentenza difinitiva del maggiore, o del minor merito: perche tanto l'Istoria dipinta da Guido, quanto quella da Domenico, sono due opere, che è un peccato, che il Tempo le maltratt sati con le sue ingiurie. Così nell'una, come nell'altra, vi sono parti assai considerabili, e qualificate, che sono bastanti à dimostrare molti documenti à quelli che haveranno ingegno d'approfittarsene; che quel questionare, val più questa in quella parte, e questa in quella qualità, è una gara così vana, che si rende ridicola appresso a chi è veramente sensato nella perfetta cognitione."

Passeris Ausführungen finden sich in einem nachträglichen Zusatz zu seiner Lebensbeschreibung des Domenichino und sind - wie dies Hess richtig bemerkt hat - bereits eine polemische Reaktion auf die Diskussionen um die "vecchiarella"Anekdote, die mit Belloris "Vite" von 1672 und insbesondere mit Malvasias "Felsina pittrice" von 1678 eingesetzt hatten. ${ }^{31}$

Malvasia (Anhang, Text 4) tut sich schwer mit der Verteidigung seines Lieblings Reni; sie kommt deutlich aus der Defensive heraus. Ähnlich wie Passeri nennt er das Urteil der Alten stümperhaft ("sciapito"). Die Authentizität der Anekdote stellt er nicht in Frage, versucht sie jedoch in seinem Sinne zu "retten“, indem er ihren Wortlaut Massani und Bellori gegenüber eigenmächtig verändert. An die Stelle des Mangels der Reaktion gegenüber dem Werk Renis, von dem die frühen Fassungen berichten, setzt er eine Reaktion besonderer Art. So spricht die Anekdote in Malvasias Schilderung von zwei unterschiedlichen Reaktionsweisen gegenüber zwei Werken, die in Bezug auf die Problematik der "affetti“ unterschiedlich konzipiert sind. Dieser Unterschied ergibt sich nach Malvasia aus dem jeweiligen Charakter der Geschichte, welche der eine oder andere Maler darzustellen hatte: $\mathrm{Da}$ es sich bei Domenichino um eine grausame Geschichte handle, habe er mit seiner Darstellung bei einem furchtsamen und erbarmungsvollen Geschlecht Mitleid erwecken müssen; weil hingegen Renis Geschichte nur sanfte und angenehme Handlungen zeige, so sei es seine Aufgabe gewesen, nur Staunen, wo nicht gar Zuversicht hervorzurufen.

Wenigstens für einen der beiden Maler kann nun 
aber die Gegenprobe vorgenommen und Malvasias Argumentation auf ihre Stichhaltigkeit hin überprüft werden. Fast zwanzig Jahre nach der Vollendung seines Frühwerks auf dem Celio erhält Domenichino die Möglichkeit, im Chorgewölbe von S. Andrea della Valle beide Szenen aus dem Leben des Heiligen gleichzeitig zu gestalten, neben der "Geißelung" auch den "Gang des Heiligen zur Kreuzigung" (Abb. 6). Obwohl Domenichino beim "Gang zur Kreuzigung", was die Gestaltung der Haupthandlung betrifft, auf Renis Lösung zurückgreift (vgl. Abb. 4), ist bei ihm die emotionale Reaktion der Zuschauer wie in der "Geißelung" außerordentlich stark: Die Mutter-KindGruppe im späteren „Gang zur Kreuzigung“ (Abb. 6) erscheint wie ein Zitat aus der früheren "Geißelungsszene" (Abb. 5).

Es kann zurückgeschlossen werden: Die Unterschiede in der Behandlung der "affetti“ durch Reni und Domenichino in ihren Fresken auf dem Celio können nicht - wie Malvasia dies tut - aus einer Verschiedenheit der Aufgabe abgeleitet werden; sie beruhen auf unterschiedlichen ästhetischen Konzeptionen der beiden Maler.

Besonders aufschlußreich an der Neufassung der Anekdote durch Malvasia ist die Tatsache, daß hier zum ersten $\mathrm{Mal}$ die Betrachtergruppe Frau und Kind deutlich mit entsprechenden, im Bild dargestellten Figuren (Abb. 10) in Bezug gesetzt wird:

"[una vecchiarella] mostrando ad un putello che seco ella aveva, una donna ivi in un angolo effigiata con un fanciullo, lodò quella e quello di un'eccessiva bellezza.“

Dabei wird, um die Analogie zu verdeutlichen, das Mädchen (,fanciulla“), das in der ursprünglichen Fassung mit der "vecchiarella" zusammen das Fresko betrachtet, in einen Buben („putello“) verwandelt. ${ }^{32}$ Diese von Malvasia neu hinzugefügte Passage geht zweifellos auf einen Brief Algardis zurück, den Malvasia in der Vita des Domenichino innerhalb seiner „Felsina pittrice“ abdruckt (Anhang, Text 2). ${ }^{33}$ Der Bildhauer Alessandro Algardi, Domenichinos früherer Vertrauter, der von Malvasia schriftlich um eine vergleichende Beurteilung der beiden Fresken gebeten worden ist, nimmt eingangs vehement für Renis Werk Stellung. Darauf werden die "chiacchere" der Alten als Märchen ("fandonie") und Erfindungen (,invenzioni“) disqualifiziert. Schließlich bringt Algardi eine zusätzliche Geschichte, die offensichtlich zur Widerlegung der "vecchiarella“Anekdote dienen soll. Noch deutlicher als in Malvasias Ergänzung zur „vecchiarella“-Anekdote ist bei Algardi der Rückbezug auf die Mutter-KindGruppe in der unteren rechten Ecke von Renis Fresko (Abb. 10): Die Figur des Knaben wird von den Müttern, die das Bild betrachten, als Modell im doppelten Sinne aufgefaßt, indem sie ihn den mitgeführten Kindern als Vorbild für anständiges Verhalten und als unerreichbares Ideal der Schönheit vorführen. Hingegen behauptet Algardi, er habe nie bemerkt, daß das Fresko Domenichinos vom Volk beachtet worden sei, oder wenn überhaupt, so habe es nur Schrecken und Grauen hervorgerufen.

Auch bei Pascoli läßt sich der Vorgang nachweisen, die Betrachterfiguren der Anekdote auf eine bildinterne Betrachtergruppe zurückzubeziehen. Wie in der ursprünglichen Fassung wird von ihm die Anekdote jedoch zum Lobe Domenichinos und als Beweis für die Wichtigkeit der "azione" in der Malerei angeführt. In seiner, der „vecchiarella“ in den Mund gelegten Beschreibung des "Geißelungs"-Freskos erwähnt Pascoli - anders als Massani und Bellori - auch die Zuschauergruppe der linken Seite und darunter im besondern das „zarte kleine Kind, das sich vor Schreck abwendet und hin zu seiner Mutter flüchtet" (Abb. 11). Unmittelbar darauf folgt ein mitleidvoller Kommentar der „vecchiarella", worin sie das Schicksal des Heiligen mit ihrem eigenen und dem des Mädchens vergleicht:

„E quel tenero bambinello tornare indietro per lo spavento, e saltare adosso alla matre? Povero santo con quanta fede, ed amore ha fissati gli occhi nel cielo! $\mathrm{Ma}$ beato lui, perchè povere siam noì figliuola mia <? !"

Aus der Abfolge der Sätze in dieser Schlußpassage aus der Bildbeschreibung Pascolis wird klar, daß hier die Mutter-Kind-Gruppe des Bildes für die Bildbetrachter wiederum als Modell dient. Doch nicht mehr die Gruppe für sich allein findet besondere Beachtung, wie dies bei Algardi der Fall war: Die Mutter-Kind-Gruppe wird als Bildelement aufgefaßt, das zwischen der dargestellten Geschichte und dem Betrachter vermittelt, indem es ihm die adäquate emotionale Reaktion auf die Historia exemplarisch vorführt. Wir möchten diesen Ansatz Pascolis im folgenden als Hypothese übernehmen und mit nochmaligem Bezug auf Reni und Domenichino präzisieren. 


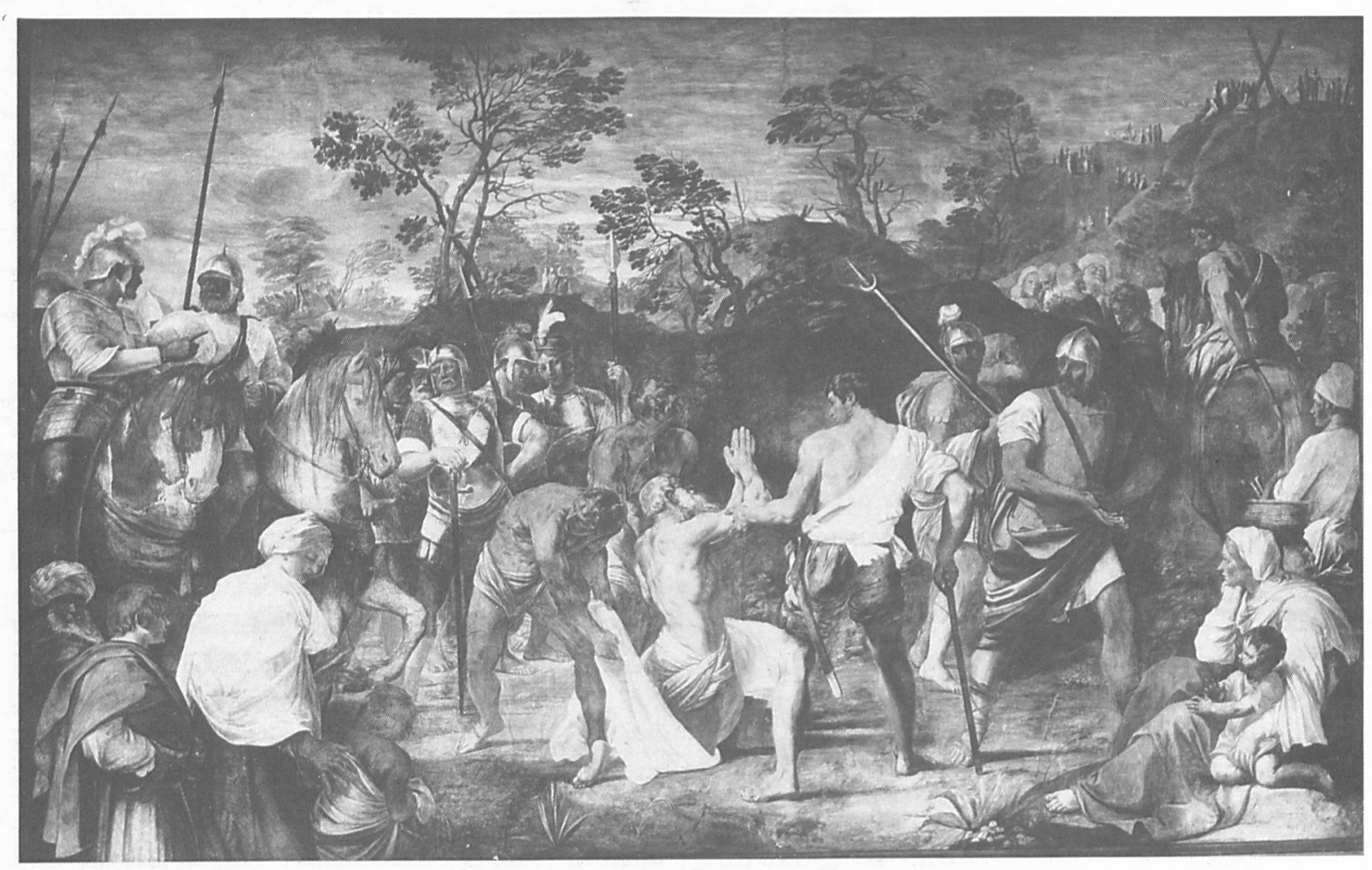

4. Guido Reni, Gang des hl. Andreas zur Kreuzigung. Rom, Oratorio di S. Andrea

\section{Das Mutter-Kind-Motiv}

Eine Geschichte des Motivs der Mutter-KindGruppe als bildinterne Betrachterfiguren ${ }^{34}$ ist unseres Wissens noch nicht geschrieben. Das Motiv wird mit besonderer Vorliebe in der barocken Historienmalerei eingesetzt, auffallend häufig im Spätbarock, wo es zu einem fast obligatorischen Bestand von Altarwerken wird. Diese Praxis geht jedoch auf eine ältere Tradition zurück. Als eines der frühesten Beispiele eines Historienbildes, bei dem die Mutter-Kind-Gruppe aus der Menge der Betrachterfiguren ausgeschieden und in doppelte „Nähe" zur Welt des Bildbetrachters gesetzt wird im Vordergrund und am Bildrand - kann Altichieros „Martyrium der hl. Katherina“ in Padua von 1384 (Abb. 7) angeführt werden. ${ }^{35}$ In einer um 1450 entstandenen Zeichnung Jacopo Bellinis mit der „Kreuztragung“ aus dem Pariser Zeichnungsbuch (Abb. 8) erscheinen Mutter und Kind in Rückenansicht und sind somit wiederum deutlich in ihrer Vermittlerfunktion gekennzeichnet. ${ }^{36}$

Ebenfalls um die Mitte des 15. Jahrhunderts skizziert Alberti in seinem Traktat "De pictura“ eine Theorie der Vermittlungsfiguren, wobei er jedoch nicht spezifisch auf die Mutter-KindGruppe eingeht. Im Kapitel über die „movimenti del corpo" gibt er den Malern, die sich die Darstellung einer Historie vornehmen, folgenden Ratschlag:

„E piacemi sia nella storia chi ammonisca e insegni a noi quello che ivi si facci, o chiami con la mano a vedere, o con viso cruccioso e con gli occhi turbati minacci che niuno verso loro vada, o dimostri qualche pericolo o cosa ivi maravigliosa, o te inviti a piangere con loro insieme o a ridere. ${ }^{“ 37}$

Bereits bei Alberti lassen sich die Aufgaben, die den Vermittlungsfiguren zugewiesen werden, den beiden rhetorischen Funktionen des docere und des movere zuordnen: Sie sollen dem Betrachter gegenüber die bildlich dargestellte Handlung erläutern und ihn zur emotionalen Teilnahme am Geschehen einladen.

\footnotetext{
5. Domenichino, Geißelung des hl. Andreas. Rom, Oratorio di S. Andrea

6. Domenichino, Gang des hl. Andreas zur Kreuzigung. Rom, S. Andrea della Valle
} 

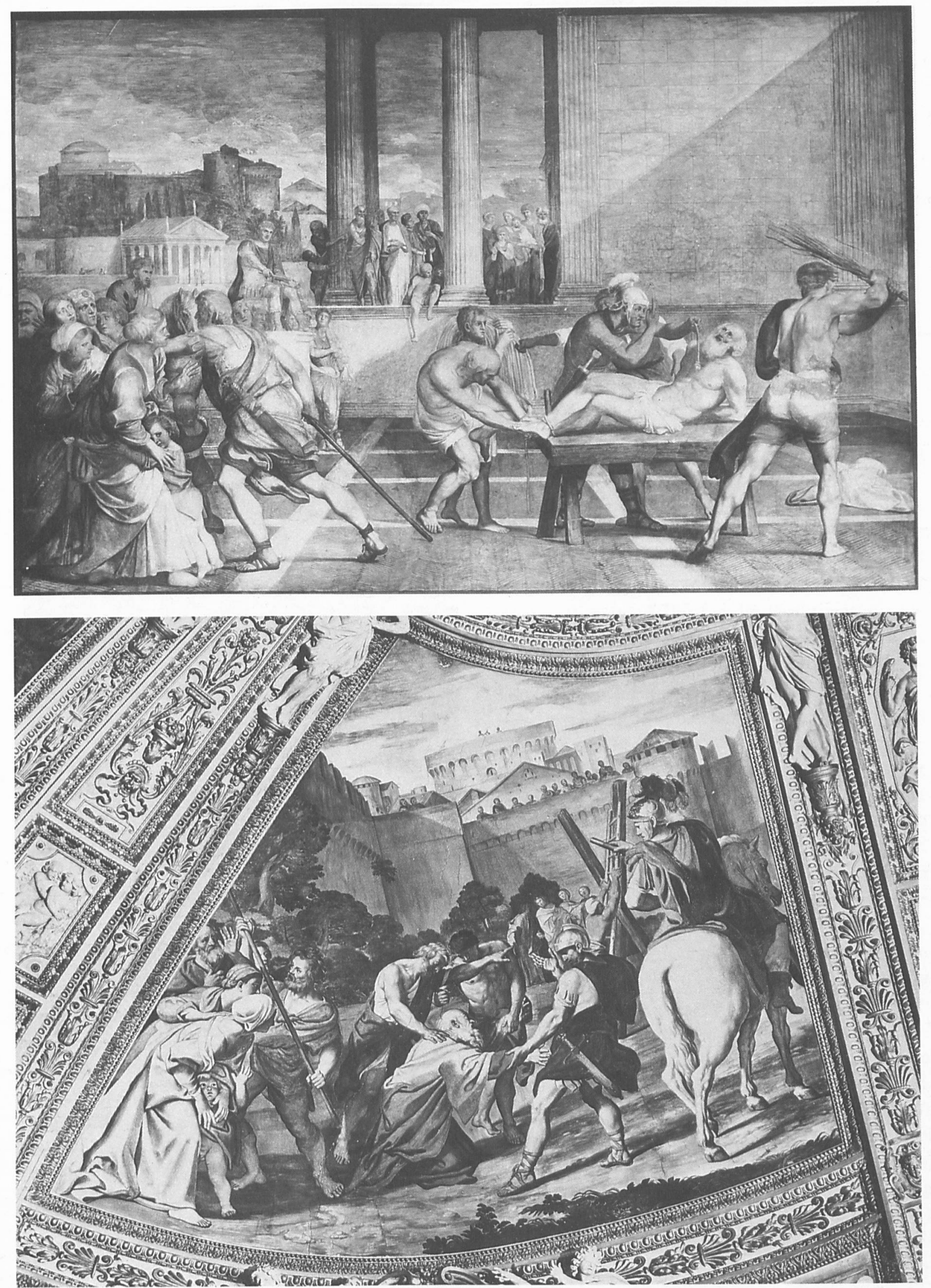


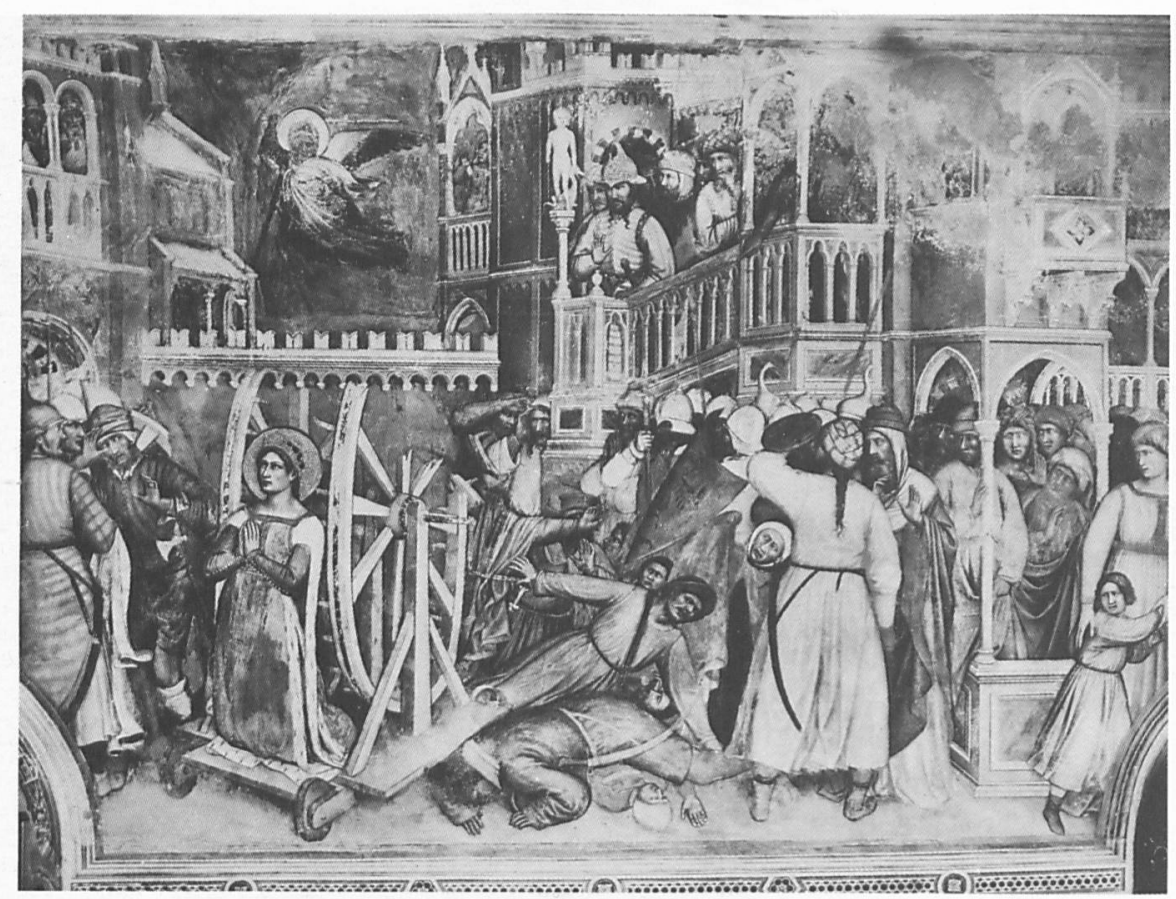

7. Altichiero, Martyrium der hl. Katherina. Padua, Oratorio di S. Giorgio

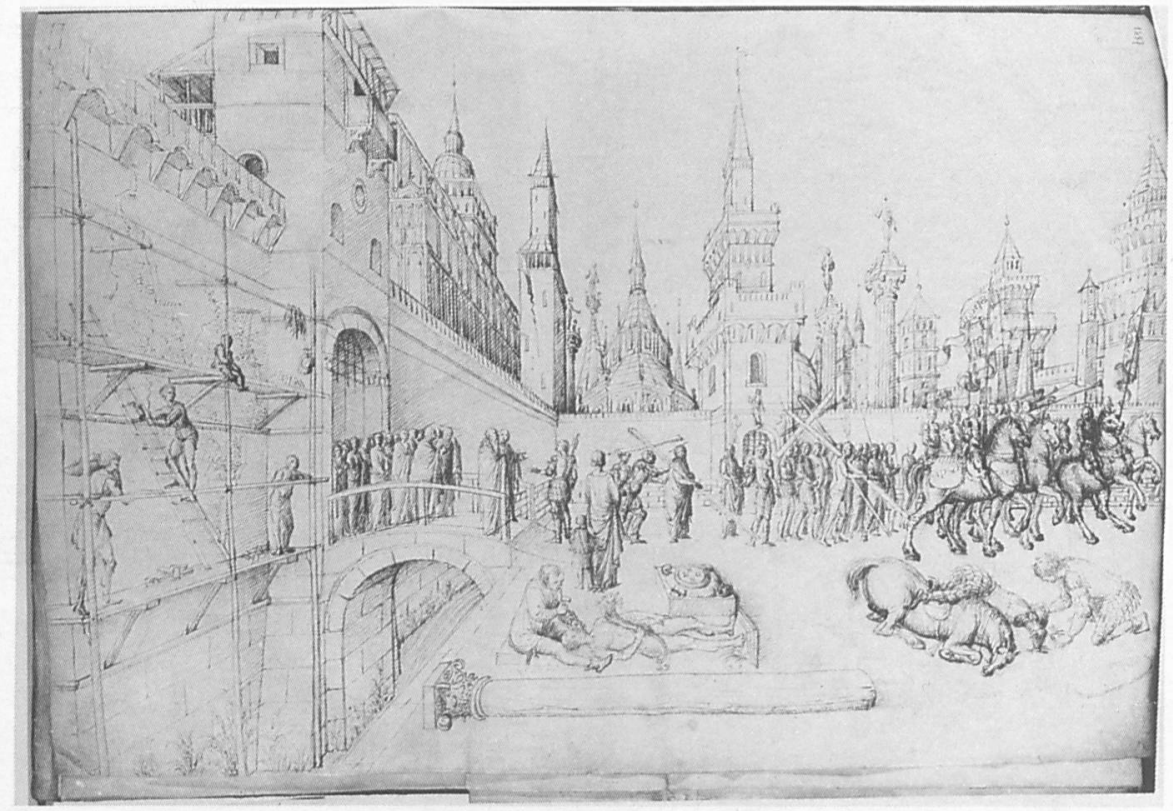

8. Jacopo Bellini, Kreuztragung. Paris, Louvre 


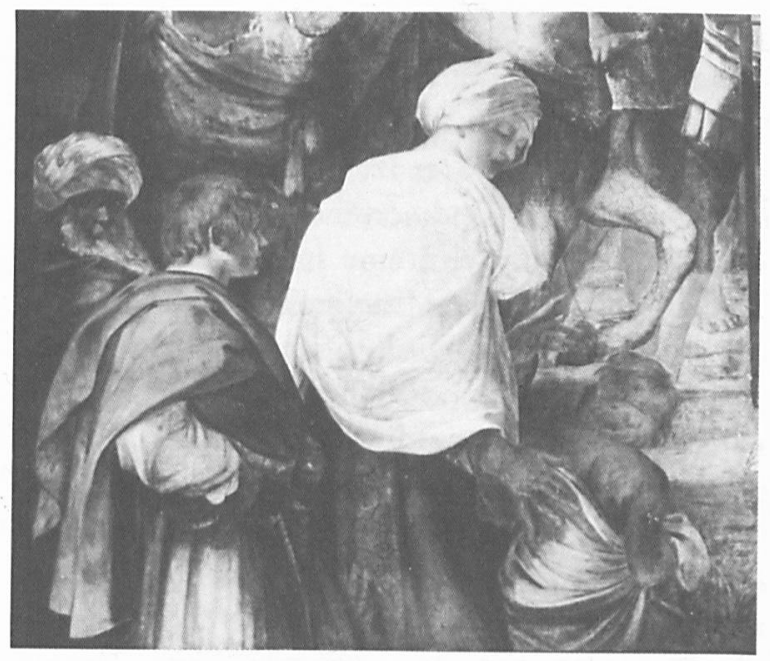

9. Guido Reni, Detail aus Abb. 4

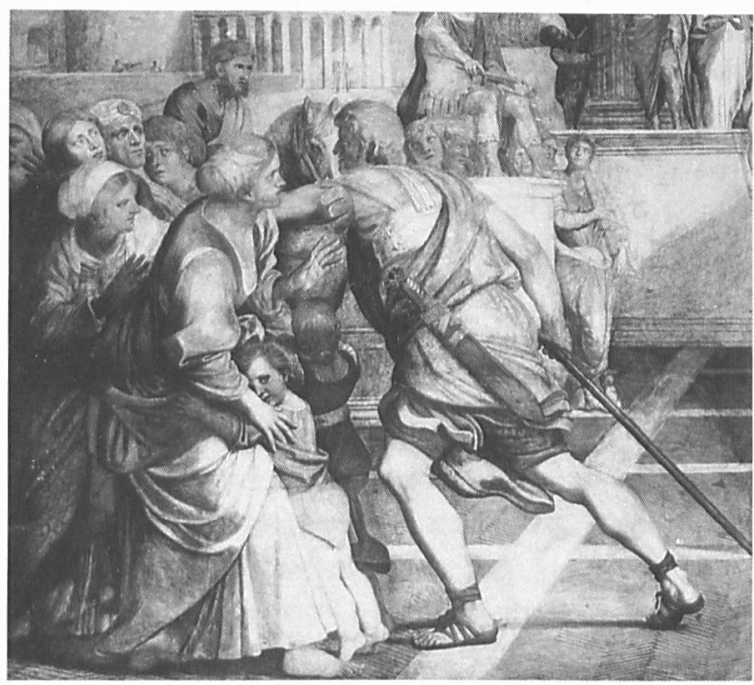

11. Domenichino, Detail aus Abb. 5

Die Tatsache, daß die Maler mit Vorliebe Mutter und Kind in der Vermittlerfunktion einsetzen, erklärt sich zweifellos daraus, daß es sich bei ihnen um affektiv besonders empfängliche Figuren handelt, wobei die interne Struktur des Mutter-KindVerhältnisses sich vor allem zur Darstellung von Reaktionen des Mitgefübls anbietet. ${ }^{38}$ Die Verdoppelung der Akteure bringt einen zusätzlichen Gewinn: Der Unterschied zwischen den jeweiligen geistigen Fähigkeiten - reine Emotivität beim Kind, vom Intellekt kontrollierte Emotivität bei der Mutter - erlaubt die Darstellung von komple-

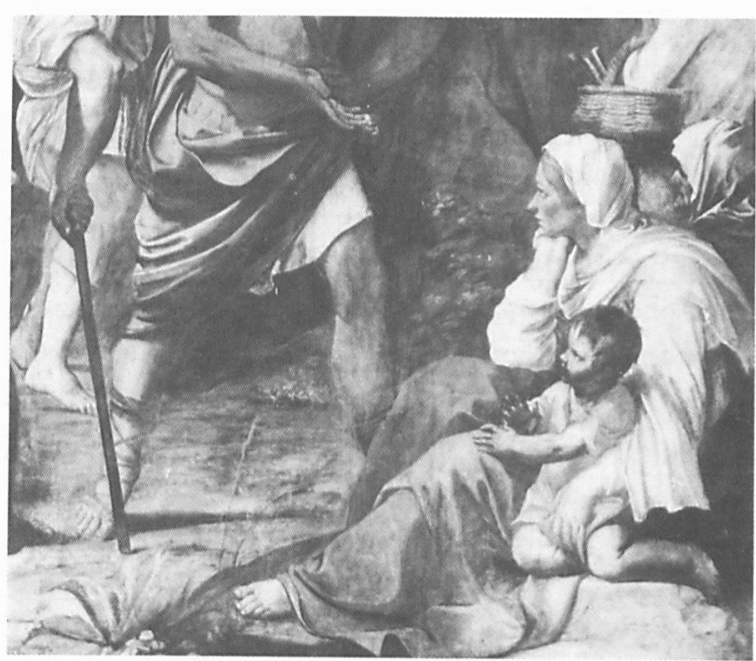

10. Guido Reni, Detail aus Abb. 4

xen Betrachterreaktionen in analytischer Form. $\mathrm{Ob}$ die christliche Theorie der Historienmalerei schon anfänglich die Anwendung des MutterKind-Motivs begünstigte, ist fraglich. Wirksam wurde die These vom Bild als Bibel für die Ungebildeten aber zweifellos bei der Reaktivierung des Motivs im Zusammenhang mit gegenreformatorischen Strömungen, wie es die Analyse der „vecchiarella"-Anekdote nahegelegt hat.

Es ist wichtig festzustellen, daß der Einsatz des Mutter-Kind-Motivs im 17. Jahrhundert nicht allgemein und undifferenziert ist, sondern von der jeweiligen ästhetischen Konzeption der Maler abhängt. So fällt auf, daß das Motiv von Caravaggio und den Caravaggisten kaum eingesetzt wird. ${ }^{39}$ In deren Ästhetik soll die dargestellte Szene auf den Beschauer direkt wirken und bedarf der Vermittlung durch bildinterne Betrachterfiguren nicht. ${ }^{40}$

Aber auch innerhalb der anti-naturalistischen Malerei, in der die Mutter-Kind-Gruppe häufig vorkommt, gibt es große Unterschiede in der Verwendung des Motivs. Sie entsprechen der jeweiligen Art und Weise, wie das Verhältnis zwischen Bildwelt und Betrachterwelt konzipiert wird. Gerade die Fresken Guido Renis und Domenichinos im Oratorio di S. Andrea verweisen auf zwei grundverschiedene Betrachterperspektiven; dies, obwohl beide Werke eine identische Inszenierung als "quadri riportati“ aufweisen. ${ }^{41}$

Reni verwendet die Mutter-Kind-Gruppe in seinem Fresko (Abb. 4) zweimal: in der linken unte- 
ren Ecke als Halbfiguren, rechts unten in der klassischen Repoussoir-Stellung. Diese doppelte Verwendung ist mit ihrer jeweiligen Funktion innerhalb der dargestellten Geschichte, dem Weg des hl. Andreas zum Kreuz zusammen zu sehen. Der Mittelgrund wird durch einen kontinuierlichen Zug der Schergen und des Verurteilten eingenommen, dem sich links vorne, von einer Art Vorderbühne aufsteigend, eine Gruppe von Mitgängern (Abb. 9) hinzugesellt. An der Spitze der Gruppe geht eine Mutter, die ihren Knaben führt und ihn gleichzeitig auf die zentrale Figur des Heiligen aufmerksam macht. Dank der Überschneidung durch den Rahmen sollen sie den Anschein erwecken, gleichzeitig der Welt des Betrachters und der Bildwelt anzugehören. Diese Art des Einsatzes von Vordergrundfiguren ist in Werken der Renaissance und des Manierismus häufig zu beobachten und findet noch in einem Altarblatt Ludovico Carraccis bei Stifterfiguren Verwendung. ${ }^{42}$

Eine andere Funktion erfüllt die RepoussoirGruppe der rechten unteren Ecke (Abb. 10). Die Gruppe von Mutter und Kind, deren Blicke ebenfalls auf den Heiligen gerichtet sind, schließt die Bildwelt an ihrer Grenze ab und trägt gleichzeitig zur Bildung des Tiefenraumes bei. Dem entspricht der Weg des Zuges: Hinter der Gruppe wendet er und bewegt sich auf das Kreuz hin, das rechts oben auf der fernen Anhöhe steht.

Bei Reni dienen die Mutter-Kind-Gruppen der Vermittlung im raum-zeitlichen Sinne: Sie sollen den Betrachter in die Bildwelt "hineinführen“, wo er eingeladen ist, die dargestellte Handlung als sequentielle Abfolge identifizierend nachzuvollziehen. Das Fehlen einer strengen zentralperspektivischen Ordnung erleichtert diese Bildwahrnehmung in der Form einer linearen Lektüre.

Ganz anders ist die Funktionsweise des MutterKind-Motivs bei Domenichino. Während bei Reni die Vermittlung zwischen Betrachter- und Bildwelt den Charakter eines "Hinein-“" und „Mitgehens" im wörtlichen, raum-zeitlichen Sinne annimmt, zielt Domenichinos Werk auf ein „SichHineinversetzen" des Betrachters im übertragenen, emotionalen Sinne hin. ${ }^{43}$

Domenichinos Fresko (Abb. 5) besitzt eine außerordentlich bewußt kalkulierte perspektivische und kompositionelle Struktur: Im Fluchtpunkt, der gleichzeitig dem Bildmittelpunkt ent- spricht, treffen sich die durch die Tempelbasis manifestierte Mittelhorizontale, die durch eine Säule angezeigte Mittelvertikale und die Diagonale der Licht-Schatten-Grenze. Dank der aus Raffaels "Borgobrand" übernommenen, gitterartigen Bodengliederung wird eine starke Raumwirkung erzielt, die jedoch im Mittelgrund durch den Tempelbau abgeblockt wird. Domenichinos strenge Raum- und Flächenordnung bewirkt einerseits, daß das Bild als eine autonome, in sich geschlossene Einheit erscheint, gleichzeitig aber auf einen fixen idealen Betrachterstandpunkt verweist. Entsprechend bleibt der reale Betrachter sich seiner vom Bild getrennten Position bewußt, und das Sich-inBezug-Setzen mit der exemplarischen MutterKind-Betrachtergruppe bekommt den Charakter eines reflektierten Prozesses.

Wichtig ist dabei, daß das Mutter-Kind-Motiv in hervorragender Stellung innerhalb einer von der Marterszene deutlich abgetrennten Betrachtergruppe erscheint. Diese Absonderung der internen Betrachterfiguren von der Haupthandlung ist durch die zurückdrängende Gestik des stockbewehrten Soldaten motiviert und entspricht dem ambivalenten Eindruck von Einbeziehung bei gleichzeitigem Getrenntsein, den der externe Betrachter dem Bildgeschehen gegenüber empfindet.

Die Diagonale der Licht-Schatten-Grenze zweifellos eine Reminiszenz an Caravaggios „Berufung des Apostels Matthäus" - besitzt ihrerseits den Charakter eines Gestus: Sie scheidet das Bild in eine Zone der actio (die unbeleuchtete Wand dient der Marterszene als dunkle Folie) und in eine Zone der reactio, worin aber auch der kommandierende Konsul seinen Platz hat (vgl. Belloris Feststellung: „si raddoppia l'azione“). ${ }^{44}$

Ein weiteres Mittel zur Verknüpfung von Bildund Betrachterwelt, der direkte Blick von Bildfiguren auf den Beschauer, wird ebenfalls von beiden Malern, aber wiederum mit unterschiedlichen Sinneffekten eingesetzt. ${ }^{45}$ Bei Domenichino trifft der erschrockene Blick des sich in den Schoß seiner Mutter flüchtenden Kindes den Blick des Betrachters (Abb. 11). In der elementaren emotionalen Reaktion des Mädchens bietet das Bild dem Betrachter den Ansatzpunkt, von dem aus er die dargestellte Geschichte rekonstruieren soll. Da er sich aber gleichzeitig seines Getrenntseins vom 
Bildgeschehen bewußt ist, wird die Rezeption des Bildes zu einem komplexen Prozeß affektiv-intellektueller Natur. Bei Reni ist es die äußerste Figur am linken Bildrand, der Turbanträger, der mit seinem Sehgestus den Betrachter einlädt, an seine Stelle zu treten und von dort aus den Weg zum Kreuze mitschreitend nachzuvollziehen (Abb. 9). Auch diese Figur dient dem Betrachter als Ansatzpunkt für die Rekonstruktion der Geschichte. Doch handelt es sich bei dem von Reni angeregten Rezeptionsvorgang um einen viel direkteren, fast ungebrochenen sinnlichen Prozeß: Der Aufbau der Handlung kommt im wesentlichen einem raum-zeitlichen Mitgehen mit den dargestellten Figuren gleich, das bis zu einem gewissen Grade mit dem primären wahrnehmenden Abtasten der Bildfläche zusammenfällt. Reni entwickelt die Historie aus der Wabrnebmungs- und Bewegungsproblematik, Domenichino aus der Affektproblematik heraus. ${ }^{46}$

Obwohl beide Maler, Reni und Domenichino, die bekannten Instrumente der Vermittlung zwischen Bildwelt und Betrachterwelt, die MutterKind-Gruppe und den Blick von Bildfiguren auf den Betrachter, einsetzen, wird dennoch jeweils an eine sehr unterschiedliche Betrachterhaltung appelliert. Nicht die Motive als solche sind bedeutungsvoll; sie werden es erst über die Art und Weise ihrer Integration in das Bildganze.

Es ist zu beachten, daß die hier skizzierte gegensätzliche Charakterisierung der beiden Werke Renis und Domenichinos vom Ausgangspunkt unserer Analyse, der "vecchiarella"-Anekdote, nicht direkt abgeleitet ist. In der Erstfassung von Massani wird Renis Fresko, weil es beim Betrachter keinerlei Reaktion hervorruft, überhaupt nicht beschrieben. Dies tut erst Malvasia, dadurch daß er eine polemische Gegenaussage Algardis durch Kontamination in die Anekdote integriert. Der zentrale Begriff in Algardis Text ist „bellezza“ ${ }^{47}$ Diese Beurteilung steht nicht im Widerspruch zu unserer Analyse: Das von Reni geforderte identifizierende "Mitgehen“ des Betrachters mit den Bildfiguren wird durch den Köder des sinnlichen Reizes zweifellos unterstützt. Der Begriff der „bellezza“ wird jedoch Reni insofern nicht gerecht, als er die komplexe narrativ-kompositionelle Struktur des Werkes und die daraus sich ergebende besondere Betrachterperspektive nicht zu fassen vermag.

\section{Zusammenfassung}

Kehren wir zur eingangs gestellten Frage nach dem interpretativen Wert der Betrachteranekdote zurück. Die Untersuchung der "vecchiarella"Anekdote hat gezeigt, daß der Wortlaut des Textes in einer primären Lektüre unergiebig, ja sogar-wie es das Beispiel Riegls gezeigt hat - irreführend sein kann. Die Anekdote als solche ist keine direkte Interpretationshilfe; sie setzt, um aussagekräftig werden zu können, ihrerseits eine interpretative Leistung voraus. Stellt man jedoch den Anekdotentext in den Kontext der zeitgenössischen Kunstliteratur, erweist es sich, daß er trotz seiner Gedrängtheit fundamentale Aspekte einer bestimmten ästhetischen Position spiegeln kann. Es ist diese Tatsache, welche im Falle der „vecchiarella"-Anekdote die Langlebigkeit und Provokationskraft des Textes erklärt und seine Eignung als Mittel der rhetorischen Argumentation ausmacht.

Die bewegte Interpretationsgeschichte der Anekdote legt Zeugnis ab vom Zusammenprall zweier gegensätzlicher Positionen, die - von verschiedenen Gruppen mit ebensolchem Ausschließlichkeitsanspruch vertreten - gleichzeitig die Ästhetik des Barockzeitalters charakterisieren. Einen übergeordneten Standpunkt einzunehmen, der es erlaubt hätte, die Werke beider Maler in ihren spezifischen Qualitäten zu würdigen, scheint den Kunstverständigen des Seicento und Settecento im allgemeinen nicht möglich gewesen zu sein. ${ }^{48}$ Der Gegensatz zwischen der Kunst Renis und jener Domenichinos kann jedenfalls nicht als eine bloße Frage der Verschiedenheit zweier "Stile" im Sinne der neueren Kunstgeschichtsschreibung gefaßt werden. In den Fresken des Oratorio di S. Andrea stehen sich zwei Konzeptionen der Historienmalerei gegenüber, die sich primär durch die Betrachterperspektive, an die sie appellieren, unterscheiden, d.h. durch die Art und Weise, wie der Betrachter mit bildinternen Mitteln aufgefordert wird, an der Rekonstruktion der dargestellten Geschichte teilzunehmen. 


\section{Anmerkungen}

Die vorliegende Untersuchung ist 1983/84 während eines Aufenthalts am Schweizerischen Institut in Rom entstanden. Sie ist dem Maler und Schriftsteller Rolf Winnewisser gewidmet, mit dem zusammen der Autor das Glück hatte, die italienischen Kunstschätze zu erkunden. Mit den Betrachterfiguren in seinem gemalten und geschriebenen Werk müßte sich eine andere Studie befassen.

1 Massanis Text ist abgedruckt bei Denis Mahon, Studies in Seicento Art and Theory, London 1947, 213-275; Mahon verweist in Anm. 54, 271 auch auf weitere Traktate des 17. und 18. Jhs., in denen die Anekdote wiederholt oder kritisch kommentiert wird. Wir geben im Anhang das Verzeichnis dieser Texte mit genauen bibliographischen Angaben und zwei zusätzlichen Verweisen. Die vier ältesten Versionen der Anekdote, die im folgenden näher behandelt werden, sind im Wortlaut abgedruckt.

2 Nach Kosch, zitiert bei Heinz Grothe, Anekdote, Stuttgart 1971, 9.

3 Ernst Kris und Otto Kurz, Die Legende vom Künstler: ein geschichtlicher Versuch, Frankfurt a.M. 1980, 32. Wir zitieren die 1934 erstmals erschienene Studie nach der neuen deutschen Ausgabe.

4 Kris und Kurz schließen zwar in ihrer Studie die Anekdoten nicht aus, welche die "Wirkung seiner Werke [i.e. des Künstlers] auf sein Publikum betreffen" (29). Aber auch die Anekdoten, die primär auf die Person des Künstlers ausgerichtet sind, bringen vielfach ebenfalls den Prozeß des Betrachtens ins Spiel. Dies ist z.B. dann der Fall, wenn der Künstler als Richter über das Werk eines Rivalen auftritt. (Zu diesen Anekdoten, als deren Urmuster die Geschichte vom Wettstreit zwischen Zeuxis und Parrhasios gelten kann, siehe Kris/Kurz 89-99). Auch bei den Anekdoten, die von Kris und Kurz in den Kapiteln III und IV besprochen werden, handelt es sich bisweilen um Betrachteranekdoten in unserem Sinne.

$5 \mathrm{Kris} / \mathrm{Kurz} 34$.

6 Es ist darauf hinzuweisen, daß auch die „vecchiarella“Anekdote, obwohl sie von Kris und Kurz nicht erwähnt wird, durchaus im Sinne der beiden Autoren behandelt werden könnte. Das Motiv der ungebildeten alten Frau als überlegene Kunstrichterin hat in der neueren Kunstliteratur den Stellenwert eines Topos. Es ist uns außer in der Anekdote, welche die Werke Renis und Domenichinos betrifft, bisher in zwei weiteren Versionen bekannt geworden: die Raffael betreffende Parallelanekdote bei Vittoria (s. Anhang 6) und eine von Diderot berichtete Geschichte (s. unter Anm. 43).

7 Unter Betrachterkompetenz verstehen wir das aktive Verfügen des Rezipienten über die Konventionen, welche ein adäquates Verständnis der Werke erst ermöglicht. Bei diesen Konventionen handelt es sich primär um die unterschiedlichen pragmatischen Attitüden, welche den "Gebrauch" des Bildwerks als Wert- oder Kultobjekt, als Gegenstand der freien ästhetischen Anschauung u.ä. bestimmen.

Wie der gleiche künstlerische Gegenstand je nach Betrachterhaltung für eine Vielzahl von Gebrauchsweisen Anlaß geben kann, hat Goethe am Beispiel der Minerva Giustiniani beispielhaft aufgezeigt (s. „Italienische Reise“, Eintrag zum 13. Jan. 1787 und Max Wegner, Goethes Anschauungen antiker Kunst, Berlin 1944, 48 f.).
Eine allgemeine Theorie der Kompetenz, aufgefaßt als modale Bestimmung des Subjektes, ist von der Pariser semiotischen Schule entwickelt worden (siehe A.J. Greimas, J. Courtés, Sémiotique: dictionnaire raisonné de la théorie du langage, Paris 1979, unter 'compétence').

8 Die Fruchtbarkeit des zweiten Weges, der Rekonstruktion der Betrachterperspektive über die Analyse bildinterner Mittel, hat Wolfgang Kemp in: Der Anteil des Betrachters, Rezeptionsästhetische Studien zur Malerei des 19. Jahrhunderts, München 1983, aufgezeigt. Vgl. auch Verf., La fonction de l'admiration dans l'esthetique du XVII ${ }^{e}$ siècle (= Documents de recherche du Groupe de recherches sémiolinguistiques 11, Paris 1980).

9 Es ist zu beachten, daß die Perspektive im engeren Sinne, verstanden als Konstruktion des Bildraumes in Funktion auf einen impliziten Betrachter, nur eines der Mittel darstellt, über die der Künstler zu Schaffung der Betrachterperspektive verfügt.

10 Wir möchten unterstreichen, daß es uns im folgenden nicht um die kaum lösbare Frage nach der "Authentizität" der Anekdote geht, etwa in dem Sinne, ob Annibale die von Massani berichtete Geschichte tatsächlich erlebt oder - was wiederum nicht das gleiche ist - so oder ähnlich erzählt hat. Die „vecchiarella" -Anekdote, erfunden oder nicht, soll dazu dienen, an einem Beispiel den möglichen interpretativen Wert der Betrachteranekdote abzuklären.

11 Die drei Oratorien, von denen die übrigen beiden der $\mathrm{Hl}$. Barbara und der HI. Silvia geweiht sind, wurden im Auftrag des Kardinals Cesare Baronio zu Beginn des 17. Jahrhunderts erneuert (S. Barbara, S. Andrea), respektive zur Erreichung einer symmetrischen Gesamtanlage neu errichtet (S. Silvia). Nach dem Tod Baronios im Jahre 1607 übernahm Kardinal Scipio Borghese das Amt des commendatore von San Gregorio. Von ihm wurde Guido Reni mit der Ausschmückung des Oratorio di S. Andrea beauftragt. Domenichino wurde die Gestaltung der Geißelungsszene überlassen. Neben den beiden Malern arbeitete überdies Lanfranco an den dekorativen Teilen der Kapelle. Obwohl eine im Innern der Kapelle über der Eingangstür angebrachte Inschrift das Jahr 1608 nennt, wurde die malerische Ausstattung erst 1609 vollendet. (Siehe Hans Henrik Brunner, "Cesare Baronio and the Convent of Gregory the Great", in: Konsthistorik Tidskrift 43 (1974) 101-120, Stephen D. Pepper, "Guido Reni's Roman Account Book“, in: The Burlington Magazine 113 (1971) 309-317, 372-386 und Richard E. Spear, Domenichino, New Haven und London $1982,155 \mathrm{ff}$ ).

12 Giovanni Battista Passeri, Die Künstlerbiographien, hrsg. von Jacob Hess, Leipzig und Wien 1934, 28 (unsere Hervorhebung). Die ganze Stelle ist aufschlußreich als Kritik der vergleichend-wertenden Kunstbetrachtung. Weniger zutreffend ist Belloris Erklärung: "Poiché questa istoria con 
l'altra di Guido ad un tempo fu discoperta ..." (s. Anhang, Text 3).

13 Zur Archäologie des Begriffs des 'Fortschritts der Künste' siehe Hans Belting, „Vasari und die Folgen“ in: Das Ende der Kunstgeschichte, München 1983, 69 ff. und die dort angegebene Literatur.

14 Giovan Pietro Bellori, Le vite, Roma 1672, 73 f. Den Höhepunkt dieser Art vergleichend-wertender Kunstbetrachtung stellt Roger de Piles' berühmte "balance des peintres", in: Cours de peinture par principes, Paris 1708, $489 \mathrm{ff}$., dar.

Aus dem Kommentar Annibales zum Streitgespräch betreffend den Vorzug von Tasso oder Ariost (,rispose che Raffaelle parevagli il miglior pittore che mai fosse stato ") wird bereits eine kritische Haltung gegenüber diesem Modus des gelehrten Diskurses deutlich. Die Frage nach dem Vorzug von Reni und Domenichino bezieht sich ebenfalls spezifisch auf die „concorrenza delle due storie a San Gregorio“. Verglichen mit der in der Vita des Domenichino berichteten "vecchiarella"-Anekdote ist das Werturteil hier im Grunde differenzierter: „rispose che Guido gli pareva il maestro e Domenico il discepolo; ma che il discepolo sapeva piú del maestro." (Vgl. auch Passeri, Ausg. Hess 1934, 29: "quando gli si dimandava del valore de' suoi Discepoli, rispondeva: Tutti si portano bene; ma Domenico è un gran furbo").

15 André Félibien, Entretiens sur les vies et sur les ouvrages des plus excellens peintres anciens et modernes, Paris ${ }^{2} 1688$, Vol. II, $320 \mathrm{f}$. Als Beweis dafür, daß Poussin das Fresko Domenichinos in S. Andrea genau studiert habe, ist sein Frühwerk „Der Triumph Davids" in Dulwich College angeführt worden. Nun hat aber Anthony Blunt, Nicolas Poussin, London 1967, 59 gerade in diesem Bild von Reni geliehene Motive ausgemacht und für die szenische Gestaltung ein überzeugenderes Vorbild nachweisen können. Vgl. dazu auch Michael Jaffé, "Poussin and Reni“, in: Festschrift Charles Sterling, Paris 1975, 213-216. Auf ein Reni-Zitat in einem anderen Frühwerk Poussins hat Stephen D. Pepper aufmerksam gemacht in: The Burlington Magazine 127 (Juni 1985), 372-74.

16 Zum persönlichen Verhältnis zwischen Reni und Domenichino während der Bologneser und frühen Römer Zeit s. Spear, Domenichino, 8-11. Die Frage, wer den entscheidenden Anteil an der Vermittlung des Auftrages für das Andreas-Fresko an Domenichino hatte, ob Reni selber, der Kardinal Borghese oder Annibale Carracci, kann nicht mit Sicherheit beantwortet werden (vgl. Spear 155).

17 Es sei hier nur auf einige klassische Einführungen in die kunstgeschichtliche Betrachtungsweise hingewiesen, in denen das vergleichende Vorgehen einen zentralen Stellenwert einnimmt: Paul Brandt, Sehen und Erkennen: eine Anleitung zur vergleichenden Kunstbetrachtung, Leipzig 1910, '1929; Matteo Marangoni, Saper vedere (zahlreiche Auflagen, auch deutsch, seit 1933); Heinrich Lützeler, Führer zur Kunst, Freiburg ${ }^{1} 1938,{ }^{4} 1941$.

18 Vereinzelte kritische Bemerkungen zur vergleichenden Methode finden sich etwa bei Hans Sedlmayr, "Zu einer strengen Kunstwissenschaft", in: Kunstwissenschaftliche Forschungen 1 (1931), 23 ff. und Die Architektur Borrominis, Berlin '1930, 62, sowic bei Otto Pächt, Methodisches zur kunsthistorischen Praxis, München 1977, 133, 251-261, 271.

19 Für eine eingehende kontrastive Beurteilung der Fresken von Reni und Domenichino nach stilistischen Kriterien siehe Luigi Serra, Domenico Zampieri detto il Domenichino, Rom 1906, 26-29. Vgl. auch Evelin Borea, Domenichino, Mailand 1965, 33-36 und $165 \mathrm{f}$.

20 Die gemeinsame Bezeichnung von Reni und Domenichino als Schüler („scolari“) Annibales bei Massani (Anhang, Text 1) und Pascoli (Text 10) ist zu präzisieren. Als Angehörige der Bologneser "Accademia degli Incamminati“ war Reni Schüler aller drei Carracci. Domenichino, der nicht vor 1595 in die Akademie eingetreten sein kann, studierte bei Agostino und Ludovico. Erst in Rom hat er Annibales Kunst zu seinem wichtigsten Vorbild erhoben, als er im Palazzo Farnese unter Annibale arbeitete und sich dessen Gestaltungsprinzipien schrittweise immer stärker aneignete. (Siehe R. E. Spear, Domenichino, New Haven und London 1982, 9 und 47-56). Im Gegensatz zu Domenichino stand Reni nie in einem direkten Gesellenverhältnis zu Annibale. Von einer besonderen Hochachtung Annibale Carraccis für Domenichino sprechen nicht nur die in der Kunstliteratur berichteten Anekdoten; Annibale hat Domenichinos Karriere durch die Vermittlung von Aufträgen aktiv gefördert (s. Spear, Domenichino, 52).

21 Beim "letterato de' primi di quel tempo", der Annibale die Frage nach dem Vorrang von Reni oder Domenichino gestellt hat, könnte Giovanni Battista Agucchi gemeint sein. Dieser, von Massani an anderer Stelle in seinem Vorwort als "persona di lettere non ordinaria" bezeichnet (s. D. Mahon, Studies, 240), war ein Vertrauter Annibales und nach dessen Tod Domenichinos wichtigster Förderer. Eine ähnliche Wahlfrage, den respektiven Vorrang von Raffael und Tizian betreffend, soll Agucchi ebenfalls Annibale gestellt haben (s. Bellori, Vite, ed. Borea, 84). Zu Agucchis Person und das Fragment seines wichtigen kunsttheoretischen Traktats, das Massani-neben der "vecchiarella“-Anekdote - im Vorwort zur Stichsammlung nach Carracci-Zeichnungen publizierte, siehe D. Mahon, Studies, $109 \mathrm{ff}$. und 240-258 (Text).

22 Paleotti, Discorso, in P. Barocchi (ed.), Scritti d'arte del cinquecento I, Mailand / Neapel 1971, 357. Zur Bedeutung der Vorstellung 'ut pictura poesis' für die neuzeitliche Theorie der Malerei siehe R. W. Lee, Ut Pictura Poesis: The humanistic Theory of Painting, New York ${ }^{2} 1967$.

23 Der Begriff 'dichiarare' umfaßt im vorliegenden Kontext eine Mehrzahl von Sinndimensionen, die deutsch nur mittels einer langfädigen Umschreibung, etwa „öffentliche, erläuternde Darlegung eines (verborgenen) Sachverhalts“, wiedergegeben werden können. Der adverbiale Zusatz "chiaramente dichiarare" hebt einzelne der erwähnten Bedeutungsdimensionen noch besonders hervor. Vgl. dazu die Charakterisierung der christlichen Historiengemälde durch Paleotti (ed. Barocchi, Scritti I, 333) als „libri per lo popolo, da leggersi publicamente per salute universale ${ }^{\alpha}$.

24 Nach Mahon, Studies, $148 \mathrm{f}$. hat Bellori seine Beschreibungsmethode von Agucchi übernommen, der sie zuvor in seinem ausführlichen Kommentar zu Annibales „Schlafender Venus" entwickelt hatte. (Agucchis Text ist von Malvasia, Felsina pittrice, Bologna 1678, Vol. I, 503-514 überliefert.) Auf eine andere Quelle für Belloris Vorgehen verweist Nicholas Turner: „Ferrante Carlo's 'descrittione della Cupola di S. Andrea della Valle dipinta dal Cavalier Gio: Lanfranchi', a source for Bellori's descriptive method", in: Storia dell'Arte 12 (1971), 297-325. Es ist jedoch zu bemerken, daß erst bei Bellori eine scharfe Trennung zwischen Handlung und Affekt vorgenommen wird. 
25 Paleotti, Discorso, ed. Barocchi, Scritti I, 334. Paleotti beruft sich hier auf Augustinus, der seinerseits eine bekannte Aussage Ciceros zu den Aufgaben der Redekunst umformt. Die Cicero-Stelle, in der docere und permovere als notwendig, delectare hingegen als fakultativ bezeichnet werden, lautet (De optimo genere oratorum I.3): „Optimus est enim orator qui dicendo animos audientium et docet et delectat et permovet. Docere debitum est, delectare honorarium, permovere necessarium".

26 Epist. XI.10, Brief an Serenus, Bischof von Marseille, zitiert nach Mon. Germ. Hist., Epist. II, 270, 14 ff. Vgl. dazu L. Gougaud, „Muta praedicatio“, Revue bénédictine 42 (1930) 168-171. Barocchi verweist in ihrem Kommentar zu Paleotti, Scritti I, 326, auf die Traktate von Gilio, Catarino, Sanders, Castellani und Molano, welche die Stelle ebenfalls zitieren. Darüber hinaus kann auf Antonio Bosio, Roma sotterranea, Rom 1650, 565 ff. hingewiesen werden. Bosio wartet im Kapitel "Dell'utilità, che si riceve dalle Sacre Imagini“ mit besonders zahlreichen Kirchenväter-Zitaten auf. Im Zusammenhang mit der "vecchiarella“-Anekdote ist eine, von Gougaud nicht aufgeführte Stelle aus einem Brief Papst Gregors II. (Epist. ad Leonem Isaurum) erwähnenswert. Darin werden als Zielpublikum ausdrücklich kleine Kinder genannt, denen von Männern und Frauen mittels Fingerzeig auf Historienbilder religiöser Unterricht erteilt wird: „Pueros parvulos nuper baptizatos in ulnis suis tenentes itemque florentes aetate iuvenes, et ex gentibus diversis profectos viri, ac mulieres, indicatis digitis historiis, eos aedificant, eorumque mentes et corda sursum ad Deum erigunt".

27 Falls bei Massani - was nicht abgeklärt werden kann - eine tatsächliche Aussage Annibale Carraccis bis in den Wortlaut hinein getreu wiedergegeben sein sollte, würde dies bedeuten, daß Annibales Konzeption der bistoria der gegenreformatorischen Theorie eng verpflichtet war. Bereits A.W.A. Boschloo (Annibale Carracci in Bologna: Visible Reality in Art after the Council of Trent, Den Haag 1974, $148 \mathrm{f}$.) hat die "vecchiarella"-Anekdote mit Paleotttis Thesen zur Funktion des religiösen Historienbildes zusammengebracht. Nach Boschloo ist die Verwandtschaft von Annibales und Paleottis Ästhetik jedoch „a matter [...] of a general affinity of vision, there is no question of a direct dependance" (146). In einem späteren Kapitel seines Buches (152 ff.) macht es Boschloo aber wahrscheinlich, daß Annibale die von Paleotti in seinem "Discorso" vertretenen Thesen zumindest indirekt gekannt hat. Vgl. dazu auch Donald Posner, Annibale Carracci, London 1971, Vol. I, 35-43.

Im Einklang mit der „vecchiarella“-Anekdote steht eine Angabe Berninis, wonach Annibale eine hohe Meinung vom kritischen Urteilsvermögen des breiten Publikums hatte (s. Paul Fréart de Chantelou, in: Journal de voyage du chevalier Bernin en France, éd. 1881, Vol. I, 384). Vgl. dazu wiederum Paleotti (bei Barocchi, Scritti I, 503), der mit Berufung auf Apelles den Ratschlag gibt, die Bilder sollten vor ihrer definitiven Aufstellung im Kirchenraum dem Volke zur Beurteilung unterbreitet werden.

28 „[...] le cose dipinte, \& imitate dal naturale piacciono al popolo, perche egli è solito à vederne di si fatte, e l'imitatione di quel che à pieno conosce, li diletta. Ma l'uomo intendente, sollevando il pensiero all'Idea del bello, che la natura mostra di voler fare, da quello vien rapito, e come cosa divina la contempla“. Zit. bei Mahon, Studies, 243. Für negative Urteile über den Geschmack des „volgo“ siehe auch - von Agucchi unabhängig - Bellori, Le vite, ed. Borea, 22.

29 S. Anhang, Text 3.

30 Wien 1908, 186. Obwohl Riegl die Anekdote nach Bellori wiedergibt, bemerkt er den Widerspruch zu dessen klassizistischer Kunsttheorie nicht.

31 Siehe Passeri, Lebensbeschreibungen, ed. Hess, 29, Anm. 1

32 Die Tendenz, die ursprüngliche Betrachtergruppe "vecchiarella“ (alte Frau) und „fanciulla“ (Mädchen) den bildinternen Mutter-Kind-Gruppen anzugleichen, läßt sich auch bei neueren Autoren beobachten. Alois Riegl (Barockkunst, 186) spricht von einem „Weib, das mit ihrem Kinde (resp. "ihrem Knaben") in die Kapelle gekommen war", und Otto Kurz leitet die Wiedergabe der Anekdote wie folgt ein: "Eine Frau aus dem Volke erklärte ihrem Kinde ... ( Reni“, Jahrbuch der kunsthistorischen Sammlungen in Wien, N.F. 11 (1937), 195).

33 Eine Darstellung von Algardis asthetischer Position gestützt auf seine Auseinandersetzung mit der "vecchiarella"-Anekdote findet sich bei Jennifer Montagu, Alessandro Algardi, New Haven und London 1985, Vol. I, 58-63. Algardi hat die Anekdote zweifellos in der von Massani überlieferten Fassung gekannt, da er für die 1646 erschienene Stichsammlung das Frontispiz entwarf.

$34 \mathrm{Im}$ folgenden abgekürzt 'Mutter-Kind-Motiv' genannt.

$35 \mathrm{Vgl}$. dazu Hanno-Walter Kruft, Altichiero und Avanzo: Untersuchungen zur oberitalienischen Malerei des ausgehenden Trecento, Diss. Bonn 1966, 95 f. Das Mutter-KindMotiv erscheint auch bereits innerhalb der Gruppe der weinenden Frauen im linken Kompartiment von Altichieros Kreuzigungsdarstellung im Santo zu Padua (s. Farbrafel S. 10 und Abb. 62 bei Gian Lorenzo Mellini, Altichiero e Jacopo Avanzi, Mailand 1965).

36 Eine genaue Analyse des Blattes aus dem Pariser Zeichnungsbuch könnte zeigen, daß die Mutter-Kind-Gruppe mit der dargestellten Geschichte hier thematisch verknüpft ist: Der Blick der den Bildbetrachter vertretenden Zuschauerfiguren richtet sich auf die Figur des kreuztragenden Christus, des Sobnes, der sich zu Maria, seiner Mutter, zurückwendet.

Die Vermittlungsfiguren in Jacopos Zeichnung lassen sich auch in Beziehung setzen zum Bibelwort "Weinet nicht über mich, weinet über euch und eure Kinder" (Luk. 23, 28). Die uns bekannten Beispiele erlauben jedoch keine schlüssige genetische Ableitung des Mutter-Kind-Motivs aus der Kreuztragungsszene. Unabhängig davon ist aber festzuhalten, daß die Lukas-Stelle die für das Mutter-Kind-Motiv entscheidende Vermittlungsfunktion, die Verknüpfung des dargestellten Ereignisses mit der Erlebniswelt des Betrachters ausspricht.

Nach den von Barbara Wilk, Die Darstellung der Kreuztragung Christi und verwandter Szenen bis um 1300, Diss. Tübingen 1969, gegebenen Verweisen sind in den ältesten Beispielen die Frauen Jerusalems obne ihre Kinder dargestellt, auch wenn - wie im Falle des Glasbildes in der Kathedrale von Bourges - das Bibelwort "Nolite flere" sogar als Spruchband in die Szene eingefügt ist. Die früheste uns bekannte Darstellung der Kreuztragung, die auf die erwähnte Lukas-Stelle Bezug nimmt und - neben zwei jugendlichen weiblichen Stifterfiguren - eine klagende Frau in Begleitung ihres Kindes zeigt, stammt mit großer Wahrscheinlichkeit aus den "Grandes Heures" des Herzogs von 
Berry; sie wäre demnach Jacquemart de Hesdin zuzuschreiben und in die Zeit zwischen 1407 und 1409 anzusetzen. (Siehe Otto Pächt, „Un tableau de Jacquemart de Hesdin?“, La Revue des Arts 6 (1956), 149-160 und Marcel Thomas, Les Grandes Heures de Jean de France Duc de Berry, Paris 1971, Farb-Abb. 110 mit Kommentar, wo bereits auf die Lukas-Stelle verwiesen wird).

37 Leon Battista Alberti, De pictura II. 42, ed. Cecil Grayson, Bari 1975, 72. In seinem "Trattato dell'arte della pittura“ wiederholt Lomazzo Albertis Ratschlag in Bezug auf die Kreuzigungsdarstellungen: „Et in questi compositioni sempre resterà estremamente lodato colui che mostrerà alcuno in atto che ti guardi piangendo, come che ti voglia dire la causa del suo dolore, \& moverti a participar della doglia sua ...". In unserem Zusammenhang ist es interessant festzustellen, daß Domenichino gerade die beiden Traktate von Alberti und Lomazzo gekannt und mit kritischer Haltung gelesen hat. (Cf. Spear, Domenichino, 26 und 29 f.)

38 Vgl. Malvasia: „Se quella del Zampieri esprime atti di crudeltà, consequentemente in un sesso timido e pietoso doveva ben promovere sensi di compassione" (unsere Hervorhebung).

39 So findet sich bei Benedict Nicolson, The International Caravaggesque Movement: Lists of Pictures by Caravaggio and his Followers throughout Europe from 1590 to 1650 , Oxford 1979, nur ein Beispiel (Nr. 233).

40 Wenn Betrachterfiguren eingesetzt werden, wie zum Beispiel in Caravaggios "Martyrium des Hl. Matthäus“, sind sie von den handelnden Figuren weder räumlich noch funktional deutlich getrennt. Dies zeigt sich in diesem Werk darin, daß bei vielen Figuren nicht sicher auszumachen ist, ob sie zur Gruppe der Schergen gehören oder zu jener der beistehenden Gläubigen. Auch in Caravaggios „RosenkranzMadonna" hat das Mutter-Kind-Motiv der linken unteren Ecke, das die zentrale Gruppe der Madonna mit dem Jesuskind auf irdischer Ebene verdoppelt, keine ausschließliche Betrachterfunktion.

41 Beide Fresken stellen mit ihren simulierten Rahmen Gemälde (nicht Tapisserien, wie Pepper angibr) dar, die mittels (gemalten) Ringen und Stricken an ebenfalls illusionistischen Pilastern korinthischer Ordnung befestigt sind (Abb. 3). Bei Renis Schema handelt es sich im Grunde um die gezähmte Variante eines manieristischen Dekorationssystems, wie es etwa Salviati im Salone des Palazzo Sacchetti angewandt hat. Vgl. Caterine Dumont, Francesco Salviati au Palais Sacchetti de Rome, Bibliotheca Helvetica Romana 12, Rom 1973, Abb. 110-116. Der "quadro riportato" kann als paradoxes Darstellungsprinzip verstanden werden, das mit den Mitteln des Illusionismus die Fiktivität und damit die Täuschungsfähigkeit der gemalten Darstellung aufzeigt. Entgegen der Information Belloris (ed. Borea, 317) war Reni und nicht Domenichino für den Entwurf des gemalten architektonischen Rahmenwerks verantwortlich (s. Spear, Domenichino, 155 und Stephen D. Pepper, Guido Reni, Oxford 1984, 224).

42 Vgl. etwa die Fresken Ghirlandaios im Chor von Santa Maria Novella in Florenz. Beim Gemälde Ludovicos handelt es sich um „Die Heilige Familie mit dem Hl. Franziskus und Stiftern" von 1591, jetzt Cento, Museo Civico. Es ist interessant, daß Scaramuccia in seinem 1674 in Pavia veröffentlichten Werk „Le finezze de' pennelli italiani“ die Funktion dieses Darstellungsprinzips in Renis Werk bereits nicht mehr versteht, wie aus seiner kritischen Bemerkung zu den zwei Figuren hervorgeht, „die ohne Notwendigkeit mehr als zur Hälfte unter der Erde stehen“ (28): „Solo in sì grand'historiato non approvò interiamente il Genio certe due Figure, che senza necessità stannosi più che mezzo sotterra ..."

43 Es ist entscheidend, Begriffe wie 'Vermittlung', 'Identifikation' und scheinbares 'Hineintreten ins Bild' jeweils genau zu präzisieren.

Der Begriff des Hineintretens im emotionalen Sinne spielt in einer Stelle aus Diderots "Salons“ eine wichtige Rolle: Zwei Darstellungen des gleichen grausamen Themas, die „Schindung des Hl. Bartholomäus", werden ganz nach der Art der "vecchiarella"-Anekdote von einer ungebildeten, alten Frau vergleichend beurteilt: „On avait exposé deux tableaux qui concouraient pour un prix proposé: e'était un Saint Barthélemy sous le couteau des bourreaux. Une paysanne âgée décida les juges incertains: Celui-ca, dit la bonne femme, me fait grand plaisir; mais cet autre me fait grand peine. [Worauf sich folgender Kommentar Diderots anschließt:] Le premier la laissait hors de la toile; le second l'y fesait entrer. Nous aimons le plaisir en personne, et la douleur en peinture“. Denis Diderot, Salons, ed. Seznec/Adhémar, Vol. II, Oxford 1963, 144.

44 Die gleiche Diagonale, diesmal in Richtung von links unten nach rechts oben dynamisiert, wird auch von Reni zur Artikulation des Bildgeschehens ausgenützt. Im Gegensatz zu Domenichino hat die Diagonale bei Reni jedoch nicht eine trennende, sondern eine verbindende Funktion: Sie leitet den Blick von der linken unteren Ecke mit der eintretenden Beschauer-Gruppe zum Bildmittelpunkt, dem Kopf und den in Anbetung erhobenen Händen des Heiligen, und gipfelt im Kreuz der rechten oberen Bildecke, dem Gegenstand der Anbetung und dem Wegziel des Zuges. (Auf die Diagonalstruktur von Renis Fresko hat bereits Hess in seiner Ausgabe von Passeris Künstlerbiographien, 86, Anm. 1, hingewiesen).

45 Zum Moriv siche Alfred Neumeyer, Der Blick aus dem Bilde, Berlin 1964.

46 Mit diesem Resultat scheinen wir uns in Widerspruch zu setzen zur gängigen Einschätzung von Renis Kunst, wonach diese vornehmlich an die Emotivität des Betrachters appelliere. Diese heutige Einschätzung Renis beruht auf den mittels Werkstattarbeiten und Drucken vielverbreiteten Einzeldarstellungen von Märtyrern und antiken Selbstmörderinnen mit ihrem charakteristischen, Raphaels Hl. Caecilia entnommenen, schmachtenden Himmelsblick. Diese Art des emotiven Appells an den Betrachter hat mit der barocken Affektdarstellung, deren eigentlicher Platz in der Historie ist, wenig zu tun. Alle Historiengemälde Renis - mit der einzigen Ausnahme des „Kindermordes" (Bologna, Pinacoteca Nazionale) - bekräftigen die anhand der Andreasszene entwickelte Charakterisierung seiner Kunst als einer Bewegungsmalerei.

47 Schon Bellori hebt neben der "gentillezza e leggiadria del penello" die "bellezza" in Renis Kunst hervor.

48 Unter den im Anhang aufgelisteten Autoren stellt allein Passeri eine Ausnahme dar. Vgl, die oben, S. 142 zitierte Stelle. 


\section{Anhang}

Verzeichnis der Texte des 17. und 18. Jahrhunderts, in denen die „vecchiarella“-Anekdote wiedergegeben ist. Die für die Untersuchung wichtigsten Fassungen sind im Wortlaut abgedruckt.

(1) MASSANI 1646 = Giovanni Atanasio Mosini (Pseud. für Giovanni Antonio Massani), Vorwort zu: Diverse figure [...] da Annibale Carracci intagliate in rame e cavate dagli originali da Simone Guilino Parigino [...], Rom 1646, 19 f. [nachgedruckt in : Denis Mahon, Studies in Seicento Art and Theory, London 1947, $270 \mathrm{ff}$.$] :$

Diuerse altre simili galanterie (Signori Virtuosi) vi sarebbono da raccontare, tanto di opere della mano, quanto di parole, e sentenze acutissime, e piene di spirito, che tutte sono inditio della finezza dell'ingegno del Carracci: ma alla presente opportunità può esser basteuol quello, che se n'è già toccato. e vi aggiugnerò solamente vn modo da lui tenuto, per lodare più vno, che vn'altro di due suoi Scolari intorno à due opere fatte da loro, delle quali, senza che io vi dia più aperta notitia di quel che farò, $\mathrm{mi}$ persuado, che ne saprete da per voi stessi ritrouare il luogo, $e$ riconoscerle, e vi riuscirà forse di gusto il sapere, che giuditio il Carracci medesimo ne facesse; e voi col vostro ben purgato, comprenderete ancora (senza che io ve ne spieghi il nome) quale delli due Discepoli venisse dal Maestro più laudato. Vn letterato de' primi di quel tempo, domandò ad Annibale, chi si fosse portato meglio di due Pittori della sua Scuola in vn lauoro, che insieme fecero per vn Cardinale, cioè vn'Historia grande per ciascuno della vita di vn medesimo Santo, dipinte à fresco in Roma dentro vna Chiesa nelle due lati, l'vn'incontro all'altro. Al quale quesito Annibale rispose, che quelle due Historie erano state cagione, che egli si era conosciuto se stesso per vn grandissimo balordo: perche non haueua mai saputo comprendere, quale di esse meritasse d'esser più lodata; sintanto che egli non imparò à conoscerlo da vna Vecchiarella; la quale hauendo per mano vna Fanciulla, si fermò vn giorno à guardare l'vna e l'altra di quelle Historie; \& egli l'osseruò; che mentre ella ad vna fissò lo sguardo, andò voltando l'occhio da ogni parte per mirarla tutta, ma non disse mai vna parola, nè diede altro segno d'alcun affetto, che in lei hauesse cagionato il guardar quella Pittura. ma poi all'altra Historia voltatasi, cominciò à dire alla Fanciulla: Vedi vedi figlia quell'huomo, che fà la tal cosa; e col dito gli accennaua la Figura, che quell'attione, ch'ella diceua, rappresentaua: e cosi di mano in mano mirando l'altre Figure, le additaua, e ne dichiaraua con gusto le attioni alla Fanciulla, la quale ancora pareua che se ne presdesse diletto. Hor vedete (disse Annibale al Letterato) com'io hò imparato à conoscere, quale delli nostri due Dipintori habbia più viuamente espressi gli affetti, e più chiaramente la sua Historia dichiarata. E questo bastò per chiarissima risposta à quel quesito.

(2) ALGARDI [spätestens 1654] = Alessandro Algardi, undatierter Brief, zit. bei C.C. Malvasia, Felsina pittrice, Bologna $1678[=(4)]$, Vol. II, $318 \mathrm{f}$ :

V.S. (egli a me scrive) lasci pur gracchiare costoro à lor posta, $l_{i}$ lasci pur dire (rispondendomi sovra le due storie fatte a concorrenza, del sudetto martirio di S. Andrea a S. Gregorio) val più la testa, le braccia el torso del S. Andrea, che adora la Croce, val più quel poco di paesetto, che non vale tutta la intiera instoria della Flagellatione, e quanto architettura vi hà mai tirato quell'altro, perche altro vi vuole che il gran studio, il gran stento, l'elaboratione, che si può conseguire da tutti. La difficoltà stà nella risolutione, nella facilità, che solo è quella che non possono, e non sanno usare se non i gran Maestri $\mathcal{E}_{c}$.

Le chiachere della vecchia, che dicono, che dicesse Annibale, che havea da lei imparato à giudicarla, sono fandonie, sono invention, io mi ci son trovato presente delle feste, E bò osservato che le madri tutte mostrando a' suoi ragazi quella bella madre, che in quella di Guido tiene il bambino, dicevano, ò che bella donna, $\grave{o}$ che bel pupo, guarda, guarda figlio come stà queto, quanto $\grave{~}$ più bello di tù, ne osservai mat che guardando dall'altra parte ne facessero caso, anzi osservai che s'inorridivano, e si attristavano à quel spettacolo; son fandonie, son baie, non si lasa dar ad intendere queste baiate $E$ c.

(3) BELLORI 1672 = Giovan Pietro Bellori, Le vite de'pittori, scultori et architetti moderni, Rom 1672; ed. E. Borea, Turin 1976, 319:

Poiché questa istoria con l'altra di Guido ad un tempo fu discoperta, concorse ciascuno a vederle come un duello di due eccellentissimi artefici, nel quale combattevano non Apelle e Protogene di una linea, ma Guido e Domenico di tutta la pittura. Volgevansi nondimeno gli occhi di tutti a Guido per la gentilezza e leggiadria del pennello, accommodato subito a piacere, ed il quale sodisfaceva piú molto che tante maravigliose parti di Domenico. Ma Annibale fra li varii discorsi altrui disse che egli aveva imparato a giudicare queste due opere da una vecchiarella, la quale riguardando la Flagellazione di Santo Andrea dipinta da Domenico, additava e diceva ad una fanciulla da essa guidata per mano: „Vedi quel manigoldo con quanta furia inalza i flagelli? Vedi quell'altro, che minaccia rabbiosamente il Santo col dito, e colui che con tanta forza stringe i nodi de'piedi? vedi il Santo stesso con quanta fede rimira il cielo?" Così detto sospirò la vecchiarella divota, e voltatasi dall'altra parte riguardò la pittura di Guido e si partí senza dir nulla. Con questo esempio insegnò Annibale in che cosa consista la perfezzione delle opere di pittura, e quanto sopra gli altri Domenico prevalesse nell'azzione e ne gli affetti che principalmente debbono attendersi in quest'arte.

(4) MALVASIA 1678 = Carlo Cesare Malvasia, Felsina pittrice, vite de pittori bolognesi, Bologna 1678, Vol. II, 17:

Fomentare [Annibale Carracci] ancora il Menichino, massime nella opposta storia del S. Andrea flagellato, sulla quale vi fù sempre il suo consiglio, ed aiuto, e che ad ogni modo riusci bensì più erudita, e studiosa dell'altra di Guido, ma non giammai così felicemente condotta. Lodavansi i vestiri decorosi, e sull'antico del Menichino, ove Guido avea imbottito certe corazze di armature, al nostro uso presente; quasi che Paolo, e dopo il Rubens, cosi bravi compositori, non avessero usato anch'essi abiti moderni, e dimestici nelle loro istorie. Ammiravano un piano mirabilmente, e con istudio manifesto digradato, per farvi ben posar le figure de'flagellanti, ove Guido (per sottrarsi, dicevano, da simile ubbidienza) avea figurato sù montagne irregolari la Croce, e l'andata di quel Santo al martirio; quasi che in una sala, ò per un'atrio s'avesse avuto ad ergere il patibolo, e far quel viaggio. Adducevano, per autentica di maggior'espressione nella Flagellazione, il testimonio sciapito di una vecchiarella, che 
accorsa anch'essa co'gli altri a vedere queste due storie, e mirando prima quella di Guido, e mostrando ad un putello che seco ella avea, una donna ivi in un'angolo effigiata con un fanciullo, lodò quella, e questo di un'eccessiva bellezza; rivoltasi poi a quella del Menichino, intenerita e compunta, cominciò a gridare della crudeltà de'manigoldi, e poco meno che a piangere; chiaro segno, diceano, che ne restò anche commossa, che non le avvenne in quella di Guido: al che avrei risposto io, che ciascuna in suo genere conseguì il suo effetto, e mosse l'affetto, come dovea. Se quella del Zampieri esprime atti di crudeltà, conseguentemente in un sesso timido, e pietoso doveva ben promuovere sensi di compassione; mà se quella del Reni non mostra che gesti mansueti e piacevoli, massime in riguardo al Santo in adorare la desiderata Croce, non doveva muovere che stupore, se non contento, e meritare in ogni figura così ben fatta quel titolo di bellezza, che non fù però dalla donna attribuita a quelle del Menichino. Se ambedue le storie avessero tolto a figurare lo stesso Santo stirato, e fieramente percosso, e che una solo di queste avesse cavato da lei tal compassione, ò allor sì che avrei detto, avesse anche mostrato maggior perfezione dell'altra.

(5) PASSERI [spätestens 1679] = Giovanni Battista Passeri, Die Künstlerbiographien, hrsg. von Jacob Hess, Leipzig und Wien
$1934,28 \mathrm{f}$.

(6) VITTORIA [1679] = Vincenzo Vittoria, Osservazioni sopra il libro della Felsina pittrice per difesa di Raffaello da Urbino, dei Carracci e della loro scuola, Rom 1703, 91-96 [Brief datiert 1679].

(7) ZANOTTI [1705] = Carlo Cesare Malvasia, Felsina pittrice, vite de'pittori bolognesi [...] con aggiunte, correzioni e note inedite del medesimo autore di Giampietro Zanotti e di altri scrittori viventi, Bologna 1841, Vol. II, $61 \mathrm{f}$. [Brief, datiert 1705].

(8) BALDINUCCI 1702 = Filippo Baldinucci, Notizie de'professori del disegno da Cimabue in qua, Florenz 1681-1728, Vol. II, 347.

(9) RICHARDSON 1722 = Jonathan Richardson, An account of some of the statues, bas-reliefs, drawings and pictures in Italy, London 1722, 320-25.

(10) PASCOLI 1736 = Lione Pascoli, Vite de'pittori, scultori ed architetti moderni, Rom 1736, Vol. II, $75 \mathrm{f}$. 